\title{
Fragility curves for non-engineered masonry buildings in developing countries derived from real data based on structural surveys and laboratory tests
}

\author{
Viviana Iris Novelli ${ }^{1,2}$ (i) $\cdot$ Raffaele De Risi ${ }^{2} \cdot$ Ignasio Ngoma $^{3} \cdot$ Innocent Kafodya ${ }^{3} \cdot$ Panos Kloukinas $^{4} \cdot$ \\ John Macdonald ${ }^{2} \cdot$ Katsuichiro Goda ${ }^{5}$
}

Accepted: 15 January 2021 / Published online: 18 February 2021

(c) The Author(s) 2021

\begin{abstract}
Malawi is located within the southern branch of the active East African Rift System, where earthquakes of moment magnitude $\left(M_{\mathrm{w}}\right) 7.0$ or greater can occur along major faults. The majority of dwellings in the country are non-engineered unreinforced masonry constructions, built by local artisans with little input from engineers. These constructions are highly vulnerable to seismic events due to poor-quality materials and lack of construction detailing. This study presents a new methodology to assess the seismic fragility curves of typical dwellings located in the Central and Southern Malawi. On-site inspections of buildings are carried out to assess geometrical and structural features of 646 façades, and an experimental campaign is performed to characterise the mechanical properties of local construction materials. The collected data allow the identification of different building typologies in terms of quality of materials and construction techniques. The critical failure modes for each of the inspected façade at their ultimate limit state are evaluated analytically. Damage limit states are defined and adopted to derive simplified Static Push-Over (SPO) curves, transformed into incremental dynamic analysis (IDA) curves by using SPO2IDA. The IDA curves are then used to obtain fragility curves for the specific damage limit states. The fragility curves presented herein are the first to be calculated for these building typologies, based on local data, and unfortunately, they show that buildings in Malawi are far more vulnerable to earthquakes than estimated from previously available international reference data. The fragility curves developed in this study may prove useful for assessing the seismic risk of these building typologies in Malawi and other East African countries.
\end{abstract}

Keywords Non-engineered unreinforced masonry buildings · Structural survey · Laboratory testing · Mechanical approach · SPO2IDA · Fragility curves

\section{Introduction}

Located in East Africa, the Republic of Malawi is a landlocked country, sharing its borders with Mozambique on the east and southwest, Zambia on the west and northwest, and Tanzania on the north and northeast. According to the 2018 Census (National Statistical Office of Malawi 2018), Malawi has a population of 17.5 million with a current growth rate of $3 \%$ per year, which is predicted to double the population by 2038 (World Bank Group Report 2018). The country has a relatively high rural population $(84 \%$ in 2018), whereas its urban population is concentrated in two major cities, Lilongwe (the capital) and Blantyre (both with

Extended author information available on the last page of the article a population of over 1 million). Being located within the East African Rift Valley, Malawi is a seismic prone country (Hodge et al. 2020), as illustrated by the recorded seismic events in Fig. 1a. The most significant earthquake in the country in recent history occurred in 1989 near Salima with $M_{\mathrm{w}} 6.3$; that caused eight fatalities and 50,000 homeless (Chapola and Gondwe 2016). The impact of such an event could have been much worse if it had hit an urban area more directly. The 2009 Karonga earthquake sequence with $M_{\mathrm{w}}$ 4.9-6.0 was the most recent major seismic sequence in the country; 300 people or more were injured, and about 4000 dwellings were destroyed or damaged (Chapola and Gondwe 2016). The economic loss was approximately $\$ 13.6$ million, and severe damage and collapse were observed, mainly in masonry buildings, which 


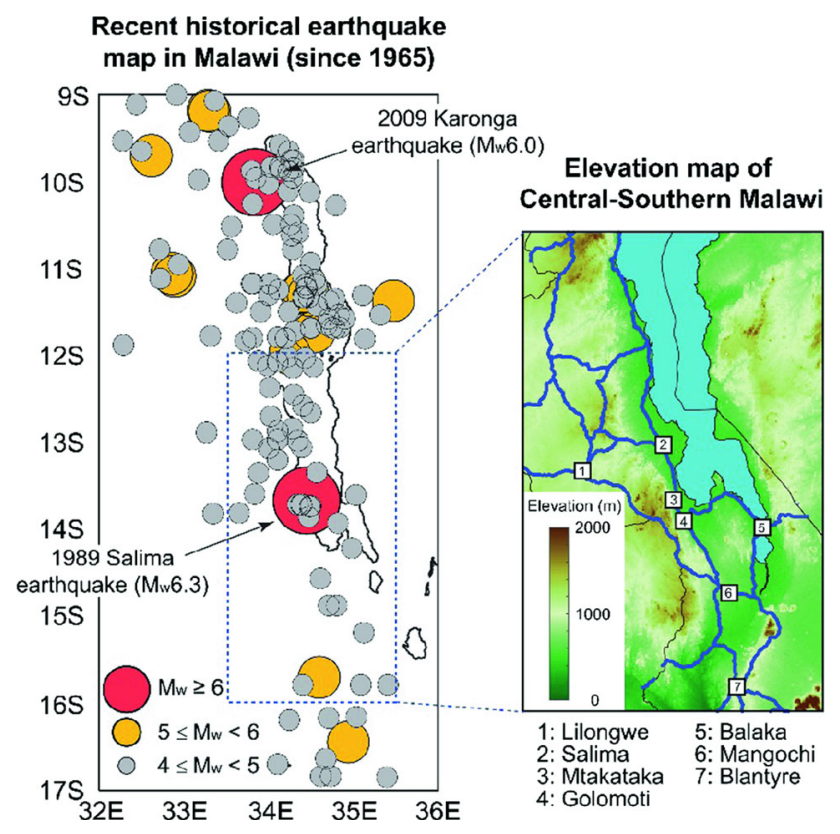

Fig. 1 a Map of Malawi, showing earthquakes since 1965; b locations of on-site surveys: urban areas of Salima and Balaka and rural villages of Lifidzi and Golomoti (Novelli et al. 2019)

are the most popular construction adopted for dwellings in many East African countries.

The official Malawian code of practice for masonry construction is MS791-1:2014 (Malawi Bureau of Standards 2014). This code is only for gravitation design, does not include provisions for seismic loads and is not usually adopted for the design of informal constructions. The code specifications require adequate construction skills and extensive economic resources. More straightforward guidelines are available for informal settlements. For example, the Safer House Construction Guidelines (Bureau TNM 2016) were developed to provide guidance on good practice to construct low-cost masonry buildings, including recommendations to reduce seismic vulnerability. Although the guidelines are a valuable reference to build safer houses, communities do not always opt for such approaches because they are not usually affordable. Therefore, houses are generally built by artisans using poor-quality construction materials and inadequate detailing, with little input from engineers (Kloukinas et al. 2020). In addition, the lack of quality control during construction leads to worsening seismic vulnerability, thus increasing the possibility of being severely damaged by future seismic events (Novelli et al. 2019). Therefore, it is imperative to develop a seismic assessment methodology that is technically sound and easy to use. Such a methodology should be tailored to Malawian constructions and should allow the level of risk to be evaluated with the aim of informing effective mitigation strategies (Novelli et al. 2019).
The primary motivation for this work is to characterise typical buildings and materials in Malawi in order to understand the local construction techniques and identify low-cost practice which can improve the building quality and reduce seismic damage in case of future earthquakes. This work also entails providing an approach capable of assessing the seismic performance and factors (e.g. material quality and structural connections) affecting structural stability, ductility, and strength of non-engineered unreinforced masonry buildings. There is another important point which should be underlined; fragility curves that are available for Malawi are from international reference sources only and are based on building characteristics inferred from neighbouring countries [e.g. data from Tanzania were used in WHE-Pager classification (Jaiswal et al. 2011)]. This underlines the need for deriving fragility curves based on local data to overcome significant bias from inaccurate information gathered from international databases. (Kloukinas et al. 2020). In this paper, seismic fragility curves are derived from Static Push-Over (SPO) analysis of mechanical models, using data gathered from a bespoke survey and obtained from structural laboratory tests conducted in Malawi. These fragility curves are provided for different buildings typologies (i.e. structural systems and quality of constructions) and different critical failure modes (i.e. Out-of-Plane and In-Plane failure modes).

In this work, the data were collected from on-site inspections that were carried out in formal and informal settlements of the urban areas of Salima and Balaka and the informal settlements of the rural villages of Lifidzi and Golomoti (Fig. 1b). These locations were selected as representative for the central region of Malawi, because of their construction practice and rapid urban growth. To identify relevant construction features impacting on the structural performance under seismic loading, 323 houses were inspected, and structural data of 646 façades (two façades for each inspected building) were collected, including typical plan, layout of openings and roof type. Every single façade was assessed by using the mechanical approach FaMIVE (Failure Mechanism Identification and Vulnerability Evaluation; D'Ayala and Speranza 2003), originally implemented for the assessment of the seismic vulnerability of historic masonry buildings, and, for the first time, used for non-engineered unreinforced masonry constructions. To adapt FaMIVE to the case study, the approach was calibrated on the data collected on-site experimentally and was employed to assess critical failure modes for each façade. The SPO curves for façade are derived by extending the work by D'Ayala (2005) assuming that the structural behaviour of façades may be governed by (1) geometric instability (D'Ayala and Paganoni 2011), (2) limited ductility (Lagomarsino 2015), or (3) 
degradation of strength (Tomaževič 2007). Thus, reflecting the uncertainty of the three types of behaviour, three SPO curves were derived for each façade, and were converted to incremental dynamic analysis (IDA) curves using the SPo2IDA tool (Vamvatsikos and Cornell 2006). SPo2IDA implements an $R-\mu-T$ relationship (reduction factorductility - period) and is also used for the seismic evaluation of existing masonry buildings in other countries (FEMA P-58-1 2012). It is worth noting that the same approach focusing on converting SPO curves into IDA curves can also be found in De Luca et al. $(2015,2018)$, where the SPo2IDA tool was applied to reinforced concrete buildings to compute explicitly the uncertainty related to the $R-\mu-T$ relationship. Finally, the IDA curves were used to derive seismic fragility curves for different building typologies and failure modes. The results provide useful data for the development of structural vulnerability evaluation tools for non-engineered unreinforced masonry structures in Malawi, and the implementation of quantitative risk assessments for East African countries.

\section{Methodology}

The proposed methodology is illustrated in Fig. 2. It consists of an approach aimed at categorising buildings in different typologies by means of steps (a) and (b) and assessing the seismic building performance by means of steps (c)-(f). Brief descriptions of the individual steps are given in the following subsections.

\subsection{On-site structural surveys}

Because of the lack of compliance to the existing guidelines, a proper inspection of structural features characterising the masonry building typologies was needed to understand local construction techniques (Fig. 2a). For each inspected building, data were collected for two orthogonal façades, noting that parallel walls of the inspected buildings typically had similar opening layouts. Data collection consisted of recording the main geometrical measurements (e.g. plan geometry, building/gable height, and opening dimensions/layout, see Fig. 3a). Information related to structural features, such as roof, masonry and mortar types, was also collected.

The inspected houses were built with locally sourced materials with poor quality control, and as a result, masonry bricks were considerably different in shape, consistency, density, and strength. For this reason, only an average brick size was measured for all inspected buildings and the quality of the structural material was classified as follows:
1. Good fabric quality: bricks (unfired or fired) have regular shapes and regular average thickness of mortar layers. Bricks (Fig. 3b1, c1) have a homogeneous texture. Brick bonding is regular. Bricks have no apparent or hair-line cracks.

2. Medium fabric quality: bricks (unfired or fired) have partially regular shapes and mortar layers. Bricks (Fig. 3b2, c2) have a medium porosity. Brick bonding is partially regular. Bricks might have light cracks or small holes.

3. Poor fabric quality: bricks (unfired or fired) have irregular shapes and mortar layers. Bricks (Fig. 3b3, c3) have a high porosity. Overlapping of bricks is irregular. Bricks often have deep cracks, possibly from poor manufacturing methods or due to weathering.

A summary of statistical analysis of the collected data is presented in Sects. 3.1.1 and 3.1.2 where descriptions of the building typology distributions and of the expected typical failure modes are provided.

\subsection{Experimental campaign on local materials}

Since local materials showed a high heterogeneity due to lack of quality control in the production phase of bricks, laboratory tests were conducted (Kloukinas et al. 2019) to determine the mechanical properties (Fig. 2b). The experiments were conducted on newly prepared specimens made of materials sourced locally, aimed at (a) replicating the actual construction practices observed during the on-site inspection and (b) characterising the strength of local materials in the country. The laboratory tests were carried out in the Civil Engineering Laboratory at the University of Malawi: The Polytechnic, in Blantyre. Test configurations consisted of:

1. Compression tests on (a) fired bricks, (b) mud mortar specimens, (c) cement mortar specimens with different cement-to-sand ratios and (d) masonry prisms. These tests aim to characterise the compressive strength of the common materials adopted in the country.

2. Tensile and shear tests on couplets and triplets, respectively. These tests were performed to determine the bonding properties between the mortar and bricks in terms of strength, cohesion, and friction.

3. Diagonal shear and Out-of-Plane flexural tests on panels. These tests were carried out to evaluate the strength and behaviour of wall panels subjected to different types of loading and to identify possible failure modes of masonry walls.

At least 6 specimens were prepared for each different test. Specimens were built using local commercially produced bricks with nominal dimensions of $200 \mathrm{~mm} \times 90 \mathrm{~mm} \times$ 


\section{BUILDING CHARACTERISATION}

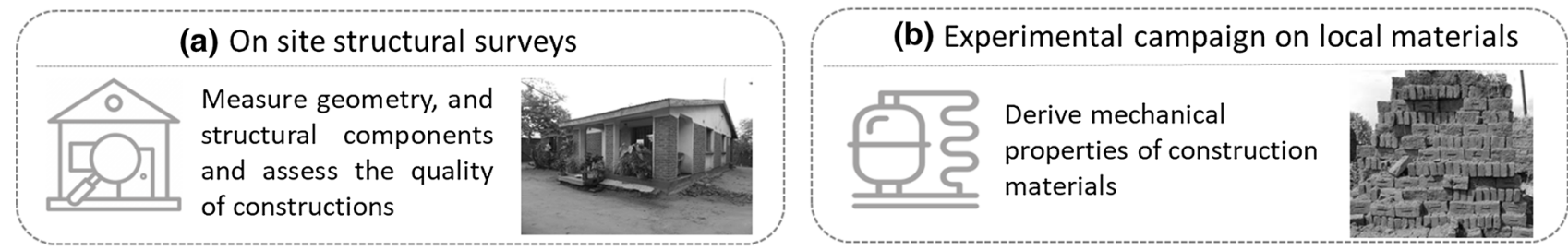

SEISMIC BUILDING PERFORMANCE

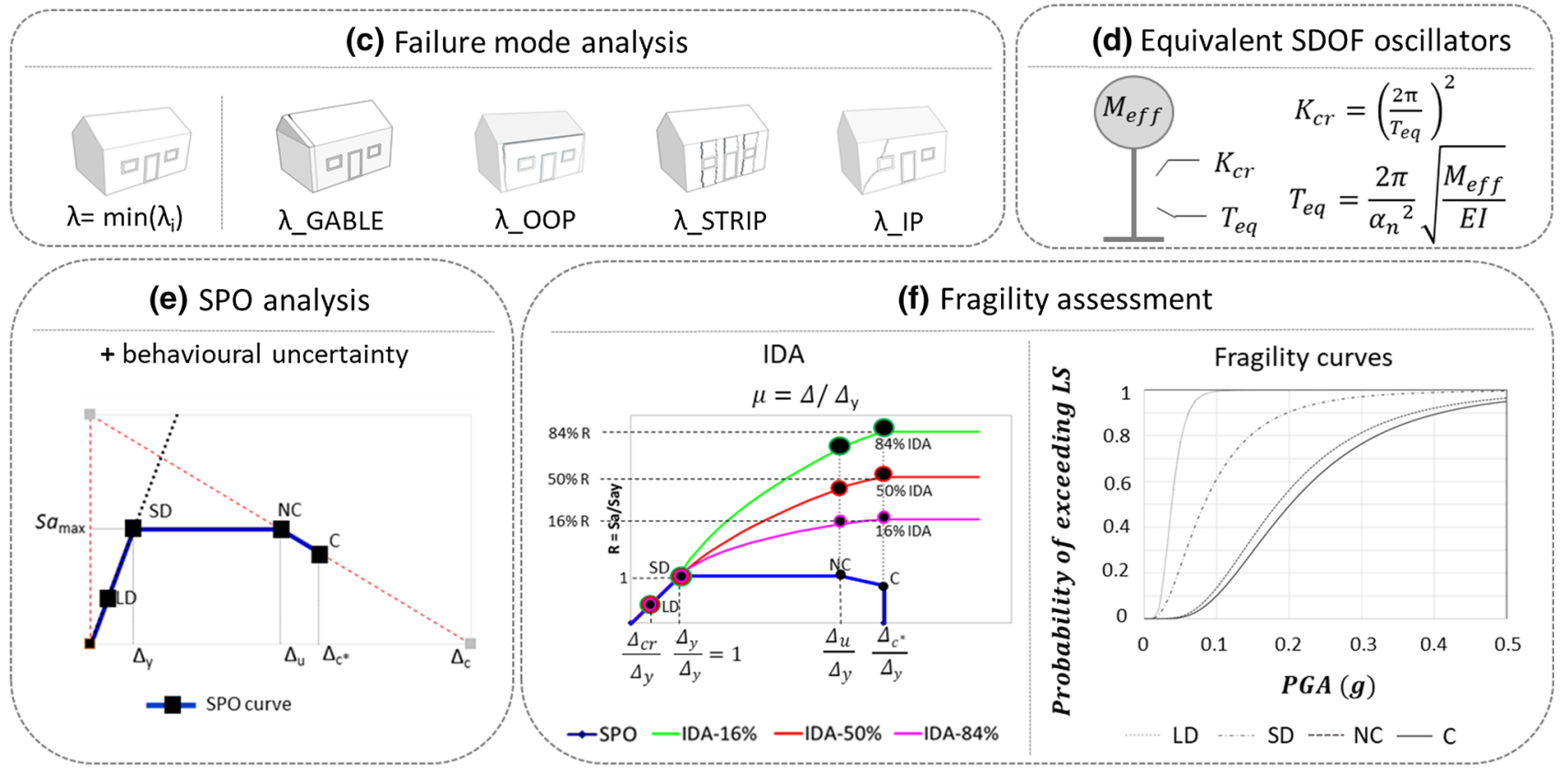

Fig. 2 Methodology for seismic performance assessment of Malawian masonry buildings

(a)

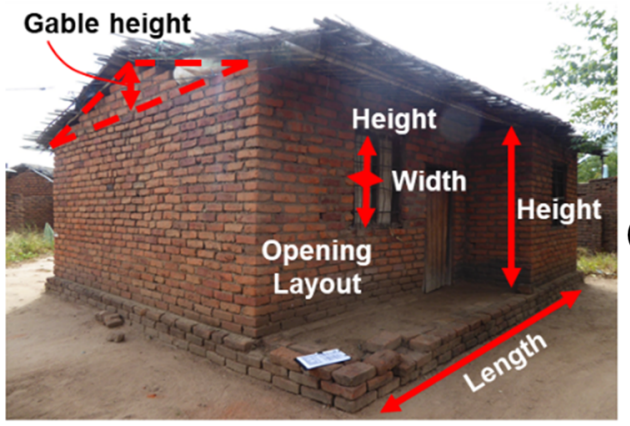

(b1)

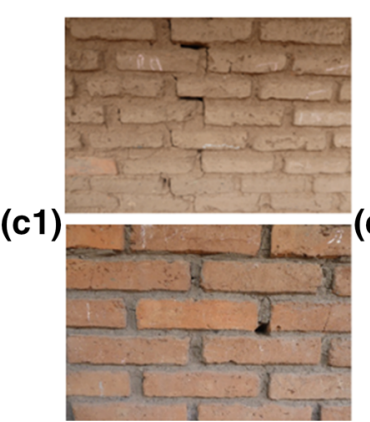

(b2)

(b3)

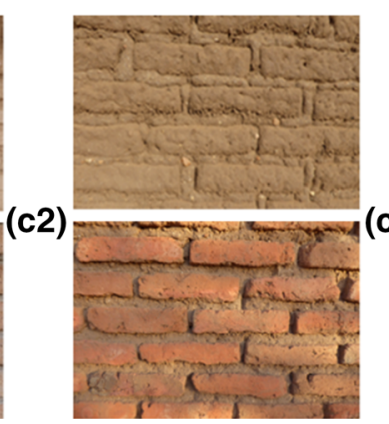

(c3)

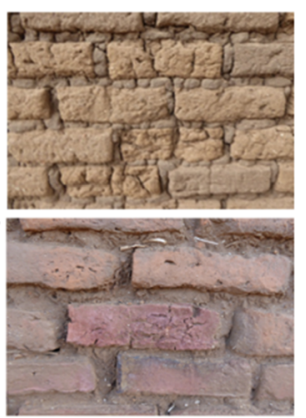

Fig. 3 a Geometrical measurements collected for each inspected façade, b1, c1 good; b2, c2 medium, b3, c3 poor fabric quality of unfired and fired bricks, respectively

$50 \mathrm{~mm}$. These bricks, although sampled from the same batch, exhibited significant variation in fabric quality (i.e. colour, shape, and size, as illustrated in Fig. 4), but this was typical of bricks observed in the field.

Two types of mortar were investigated: (1) mud, which is a common material for houses with single-/double-skin walls characterised by fabric quality varying from poor to medium, and (2) cement, mostly used for houses with double-skin walls characterised by fabric quality varying from medium to good. For cement mortar, different cement-to-sand ratios were employed: 1:4; 1:6 and 1:8, where the first two are the values recommended by MS7911 (Malawi Bureau of Standards 2014) and the Safer House Construction Guidelines (Bureau TNM 2016), respectively, 
(a)

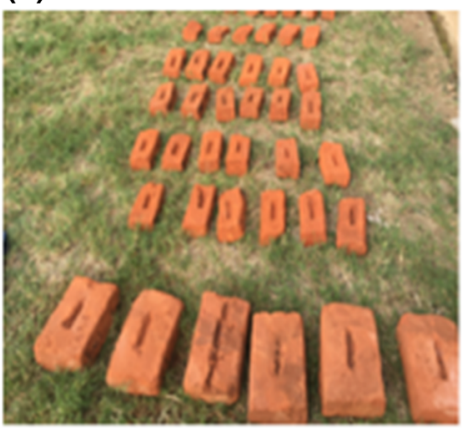

(b)

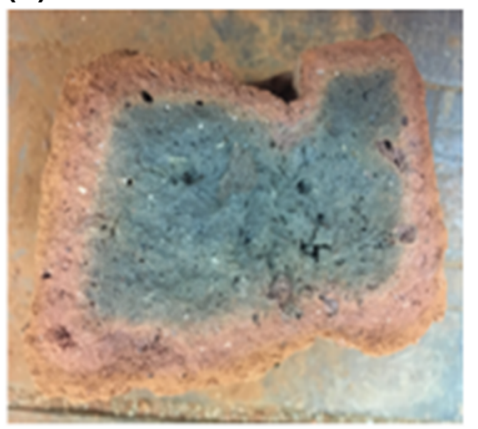

(c)

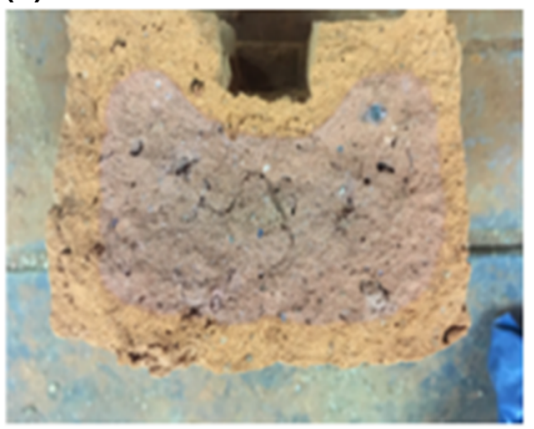

Fig. 4 Brick specimens that are used for the material tests

while the last one is commonly adopted by local artisans since more affordable.

The cement mortars adopted for the prisms and panels were prepared in two conditions: (a) "Unfavourable", by applying mortar on dry and dusty bricks (simulating common in situ conditions) and (b) "Favourable", with bricks soaked in water prior to masonry construction (recommended conditions). Hereafter, a letter " $U$ " or "F" is used to indicate the bonding conditions of the mortar, e.g. 1:4F. The equipment used for the experiments consisted of testing rigs fixed on the laboratory's strong floor, a commercial vision-based system (Imetrum Video Gauge, Bristol, UK) (https://www.imetrum.com/) for measuring displacements, and synchronised analogue load cell signals for measuring applied loads. Results of the experimental campaign are summarised in Sect. 3.2.

\subsection{Failure mode analysis}

During recent decades, a wide range of methods and techniques have been implemented to assess the seismic performance of masonry buildings, ranging from advanced numerical modelling aimed at capturing the 3D seismic behaviour of structures, to simplified approaches based on the calculation of collapse load factors. Methods of higher complexity typically rely on finite element methods (Vlachakis et al. 2019; Dumaru et al. 2020). These approaches allow reliable simulation of building performance using numerical models based on detailed geometry, structural features, and mechanical material properties. Notwithstanding issues in capturing complex behaviour and failure modes of masonry structures, these approaches are broadly used in the engineering communities. Most of them are intended for In-Plane analysis, while only a small fraction of them is applicable to both In-Plane and Out-of-Plane analyses (Novelli et al. 2019). Among the analytical approaches that are suitable for assessing the seismic vulnerability of masonry constructions, mechanical models have the advantage of evaluating the structural performance of masonry buildings for both In-Plane and Out-of-Plane failure modes (D'Ayala and Speranza 2003; Addessi et al. 2014; Giordano et al. 2020; Preciado et al. 2020). These methods aim to estimate collapse load factor multipliers of a given configuration of macro-elements by imposing kinetic energy equations and have the benefit of needing fewer input parameters than finite element methods.

In this study, the failure mode analysis (Fig. 2c) is carried out using FaMIVE, originally developed by D'Ayala and Speranza (2003) to assess the vulnerability of historic masonry buildings, and subsequently expanded by Novelli and D'Ayala (2012) and Novelli et al. (2015). FaMIVE calculates the strength of the masonry starting from cohesion and friction contact interfaces and can assess the effect of irregular opening layouts on the seismic performance of masonry buildings. This approach assumes that a single wall behaves as an assemblage of macroelements held together by compressive forces. For the specific context of Malawi, these forces are estimated for single façades by considering geometrical/structural features, connections to adjacent walls, loading and restraint effects of horizontal structures (i.e. roof/floors) observed on-site (Novelli et al. 2019). Materials and related mechanical properties are characterised by defining the quality of the fabric for each inspected façade. The friction and cohesion are defined with reference to the experimental results of Kloukinas et al. (2019) reported in Sect. 3.2.

FaMIVE allows the identification of the most vulnerable failure mode for each façade by performing failure mode analyses based on the principle of Virtual Work. Specifically, the procedure allows the collapse load factor multiplier $(\lambda)$ to be calculated for each failure mode, that is the non-dimensional ground acceleration amplitude that activates the failure mode. The factor $\lambda$ is calculated for each of the failure modes deemed plausible on the basis of the post-earthquake assessment carried out after the Karonga earthquake in 2009 (see Sect. 3.1.2). Among the computed collapse load factor multipliers, the failure mode with the 
smallest multiplier is considered to govern the collapse of the building, i.e. critical failure mode.

\subsection{Equivalent single-degree-of-freedom (SDOF) oscillators}

An equivalent SDOF oscillator is derived for each inspected façade (Fig. 2d). The SDOF oscillator has a lumped mass $M_{\text {eff }}$, equal to the effective mass involved in the failure mode (i.e. in-plane or out-of-plane) estimated as a critical one using FaMIVE (D'Ayala and Speranza 2003). The equivalent natural period $T_{\text {eq }}$ of the single SDOF oscillator is calculated by the following expression (Chopra 2000):

$T_{\text {eq }}=\frac{2 \pi}{\alpha_{n}{ }^{2}} \sqrt{\frac{M_{\text {eff }}}{E I}}$

where $\alpha_{n}=1.88$ for an equivalent cantilever, $E$ is the homogenised modulus of elasticity of the masonry wall, and $I$ is the second moment of area of the façade (i.e. $\frac{L_{\mathrm{tot}} \cdot t^{3}}{12}$ for Out-of-Plane mode and $\frac{t \cdot L_{\text {tot }}^{3}}{12}$ for In-Plane failure mode where $L_{\text {tot }}$ and $t$ are the total length and thicknesses of the wall, respectively).

The maximum strength of each SDOF oscillator failing In-Plane or Out-of-Plane is expressed in terms of maximum spectral acceleration $\left(\mathrm{Sa}_{\mathrm{o}}=\frac{g \lambda}{e^{*}}\right)$ where $\mathrm{Sa}_{\mathrm{o}}$ is a function of the minimum collapse load factor multiplier $\lambda, g$ is the gravitational acceleration, and $e^{*}$ is the ratio between $M_{\text {eff }}$ and the total lumped mass of the SDOF oscillator equivalent to the total mass of the single façade (Tomaževič 2007; D'Ayala and Novelli 2014; Sorrentino et al. 2017).

The collapse displacement $\Delta_{\mathrm{c}}$ of each SDOF oscillator is defined as the In-Plane or Out-of-Plane displacement causing the collapse. This displacement is assumed equal to either $t$ or $l / 2$, where $l$ is the masonry brick length, depending on which of the two dimensions is more critical (D'Ayala and Paganoni 2011). Once the equivalent SDOF oscillator is characterised, the spectral acceleration $\mathrm{Sa}_{\mathrm{o}}$ and the collapse displacement $\Delta_{c}$ are adopted to define the bilinear $\mathrm{Sa}_{\mathrm{o}}-\Delta_{\mathrm{c}}$ relationship describing the collapse of the façade (Fig. 2e).

\subsection{Static pushover analysis and behavioural uncertainty}

Many factors related to the data collected on-site (e.g. quality and type of masonry, mortar type, wall thickness, and connections) affect the building performance under seismic events, and uncertainties on these factors can lead to a different behaviour of the building performance. Such uncertainties are taken into account by assuming that the critical failure mode identified by FaMIVE may occur in three different ways: (a) geometric instability (D'Ayala and Paganoni 2011), (b) limited ductility (Lagomarsino 2015), and (c) degradation of strength (Tomaževič 2007) (see Fig. 5).

Three SPO curves are obtained for each façade to quantify the behavioural uncertainty and determine the effects of this uncertainty in the building vulnerability assessment. The SPO curves (i.e. the thick lines derived for façades failing in Out-of-Plane failure mode and the dashed blue lines derived for façades failing in In-Plane failure modes in Fig. 5) correspond to tri-linear (or bi-linear) models obtained from the bi-linear $\mathrm{Sa}_{\mathrm{o}}-\Delta_{\mathrm{c}}$ relationship through the definition of four points (i.e. black square markers in Fig. 5) corresponding to four Limit States: (LS): (i) Light Damage (LD), (ii) Severe Damage (SD), (iii) Near Collapse (NC), and (iv) Collapse $(C)$.

The match between the bi-linear $\mathrm{Sa}_{\mathrm{o}}-\Delta_{\mathrm{c}}$ and the SPO tri-linear (or bi-linear) models is based on existing mechanical approaches (Doherty et al. 2002; D'Ayala 2005; Derakhshan et al. 2014; Lagomarsino 2015), relying on the assumption that masonry walls behave as rigid bodies, which can collapse in In-Plane failure modes or rock in Out-of -Plane failure modes about the pivot points positioned at cracks. According to these approaches, the threshold resistance measured in experimental SPO tests (Griffith et al. 2004) for walls failing in In-Plane failure modes correspond to the values of $\mathrm{Sa}_{\mathrm{o}}$. Conversely, for wall failing in Out-of-Plane failure modes, these deform significantly, when subjected to high pre-compression, and therefore the threshold resistance is significantly reduced, as illustrated in Fig. 5, to $\mathrm{Sa}_{\max }$, as indicated in the experimental data (Griffith et al. 2004).

\subsubsection{Light damage}

Indicated in Fig. 5 by $\operatorname{LD}\left(\Delta_{\mathrm{cr}}, \mathrm{Sa}_{\mathrm{cr}}\right)$, this limit state is reached when the first crack occurs (Tomaževič 2007). Based on the experimental results (Griffith et al. 2004) and numerical modelling (Doherty et al. 2002; Derakhshan et al. 2014; Lagomarsino 2015), the first crack defines the first branch of the SPO curve from the origin up to LD. This branch has a gradient of $\delta_{\mathrm{cr}}$ equal to $\left(2 \pi / T_{\mathrm{eq}}\right)^{2}$, resulting from the initial elastic behaviour of the equivalent SDOF oscillator in an ADRS (Acceleration Displacement Response Spectra) domain. The crack initiation point on the first branch of the simplified SPO curve is determined according to D'Ayala and Paganoni (2011) by the cracking displacement: $\Delta_{\mathrm{cr}}=\frac{\mathrm{Sa}_{\mathrm{cr}}}{4 \pi^{2}} T_{\mathrm{eq}}{ }^{2}$ and the corresponding cracking acceleration: $\mathrm{Sa}_{\mathrm{cr}}=\operatorname{tg} / 4 H_{\mathrm{tot}}$, where $H_{\text {tot }}$ is the total height of the wall. 


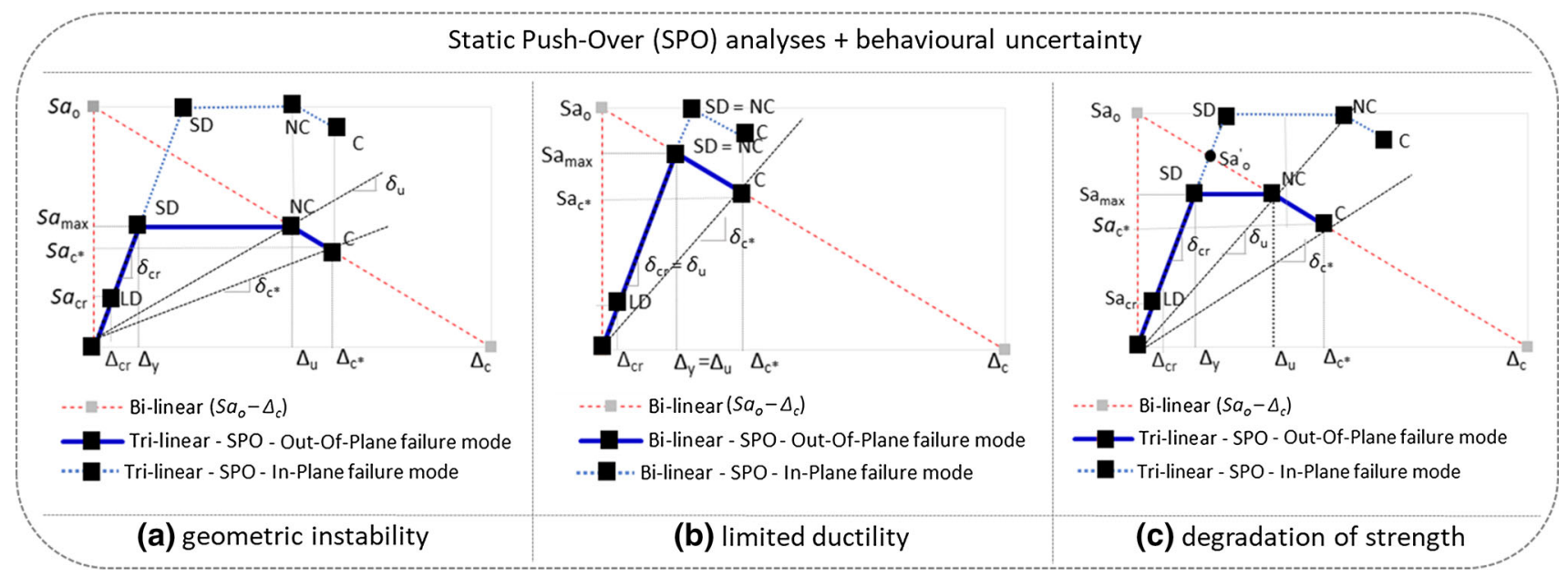

Fig. 5 Static pushover curves corresponding to the three different behaviours: a geometric instability, b limited ductility, c degradation of strength. Note $\mathrm{Sa}_{\max }=S a_{y}=\mathrm{Sa}_{\mathrm{u}}$

\subsubsection{Severe damage}

Indicated in Fig. 5 by $S D\left(\Delta_{y}, \mathrm{Sa}_{y}\right)$, this limit state corresponds to the first yielding of the façade. This limit state has the yielding spectral acceleration $\left(\mathrm{Sa}_{y}\right)$ equal to $\mathrm{Sa}_{\max }$, the spectral acceleration defined in Sect. 2.5.3 for all three possible structural behaviours of the façade. Once the value of $\mathrm{Sa}_{\max }$ is calculated, $\Delta_{y}$ is calculated as $\mathrm{Sa}_{\max } / \delta_{c r}$.

\subsubsection{Near collapse}

Indicated in Fig. 5 by $\mathrm{NC}\left(\Delta_{\mathrm{u}}, \mathrm{Sa}_{\mathrm{u}}\right)$, this limit state corresponds to the attainment of the façade's critical failure mode identified by FaMIVE. Different values of $\Delta_{\mathrm{u}}$ are defined for each structural behaviour, while $\mathrm{Sa}_{\mathrm{u}}$ is defined differently according to the critical failure mode. In particular, for In-Plane failure modes, the value of $\mathrm{Sa}_{\mathrm{u}}$ coincides with the value of the spectral acceleration $\mathrm{Sa}_{\mathrm{o}}$ at failure (see Sect. 2.4). This agrees with the existing literature related to In-Plane loaded unreinforced masonry walls (Portioli et al. 2013; Casapulla and Argiento 2018). For Out-of-Plane failure modes, $\mathrm{Sa}_{\mathrm{o}}$, which activates the rocking failure mode, is reduced to $\mathrm{Sa}_{\mathrm{u}}$, as proposed by the tri-linear models of Doherty et al. (2002) and Lagomarsino (2015). This reduction from $\mathrm{Sa}_{\mathrm{o}}$ to $\mathrm{Sa}_{\mathrm{u}}$ depends on three different types of structural behaviour as follows:

\section{(a) Geometric instability (Fig. 5a)}

According to D'Ayala (2005), the critical failure mode for geometric instability is determined by the ultimate displacement $\Delta_{\mathrm{u}}$. For In-Plane and Out-ofPlane failure modes, the displacement $\Delta_{\mathrm{u}}$ is equal to $\min (s / 2, t / 3)$ respectively, where $s$ is the overlap between two bricks (D'Ayala and Novelli 2014). $\mathrm{Sa}_{\mathrm{u}}$ is found from $\Delta_{\mathrm{u}}$, being on the bi-linear $\mathrm{Sa}_{\mathrm{o}}-\Delta_{\mathrm{c}}$ curve (Doherty et al. 2002) and the linear system with gradient $\delta_{u}=\mathrm{Sa}_{\mathrm{u}} / \Delta_{\mathrm{u}}$, and the bi-linear $\mathrm{Sa}_{\mathrm{o}}-\Delta_{\mathrm{c}}$ curve (Doherty et al. 2002; Fig. 5a). All SPO curves derived by this approach are tri-linear and have $\mu=\frac{\Delta_{\mathrm{u}}}{\Delta_{\mathrm{v}}} \geq 1$.

(b) Limited ductility (Fig. 5b)

In agreement with the bi-linear force-displacement model of Lagomarsino (2015) based on the hypothesis that masonry walls have limited ductility, the ultimate limit state is defined by setting the ultimate displacement $\Delta_{\mathrm{u}}$ equal to $\Delta_{y}$. $\mathrm{Sa}_{\mathrm{u}}$ for façades vulnerable to Out-of-Plane failure modes is taken equal to the intersection between the linear system with gradient $\delta_{\mathrm{u}}=\delta_{\mathrm{cr}}$, and the bi-linear $\mathrm{Sa}_{\mathrm{o}}-\Delta_{\mathrm{c}}$ curve. All SPO curves derived by this approach are bi-linear and have $\mu=\frac{\Delta_{u}}{\Delta_{y}}=1$.

(c) Degradation of strength (Fig. 5c)

Following Tomaževič's model (2007), the ultimate limit state is defined as the point when the actual resistance of the wall system degrades to $80 \%$ of the maximum strength on the SPO curve. This hypothesis for the degradation agrees with much of the experimental evidence from the last three decades (El-Dakhakhni et al. 2013; Chourasia et al. 2016) and various numerical modelling (Salmanpour et al. 2013; Diaz et al. 2019). According to this hypothesis, for façades failing in Out-of-Plane failure modes, $\mathrm{Sa}_{\mathrm{u}}$ is given by reducing $\mathrm{Sa}_{0}^{\prime}$ by $20 \%$, where $\mathrm{Sa}_{0}^{\prime}$ is the intersection between the linear system with gradient $\delta_{\text {cr }}$ and the bi-linear $\mathrm{Sa}_{0}-\Delta_{\mathrm{c}}$ curve. The ultimate displacement $\Delta_{\mathrm{u}}$ for wall systems failing in In-Plane failure modes is calculated as in case (a), while for wall systems failing in Out-of-Plane modes, $\Delta_{\mathrm{u}}$ is taken as the displacement at the 
intersection between the linear system with gradient $\delta_{\mathrm{u}}$ and the bi-linear curve $\left(\mathrm{Sa}_{\mathrm{o}}-\Delta_{\mathrm{c}}\right)$. All SPO curves derived by this approach are tri-linear and have $\mu=\frac{\Delta_{\mathrm{u}}}{\Delta_{\mathrm{y}}} \geq 1$.

\subsubsection{Collapse}

Indicated in Fig. 5 by $C\left(\Delta_{c^{*}}, \mathrm{Sa}_{c^{*}}\right)$, this limit state is defined identically for all three considered types of structural behaviour. Specifically, $\mathrm{Sa}_{\max }$ is decreased by $20 \%$ to $\mathrm{Sa}_{c^{*}}$. The additional degrading branch is defined to capture the extra safety ductility of the wall (Tomaževič 2007). $\Delta_{c^{*}}$ represents the displacement related to $20 \%$ drop in spectral acceleration. This is directly defined from $\mathrm{Sa}_{c^{*}}$, as it is located on the bi-linear $\mathrm{Sa}_{\mathrm{o}}-\Delta_{\mathrm{c}}$ curve and linear system with a gradient of $\delta_{c *}=S a_{c^{*}} / \Delta_{c^{*}}$.

\subsection{Fragility assessment}

The SPO curves can be transformed, via SPO2IDA (Vamvatsikos and Cornell 2006), to IDA curves for the derivation of fragility curves (Fig. 2f). Such a procedure is currently adopted in FEMA P-58-1 (2012) for the seismic assessment of buildings in the USA and is regarded as applicable to masonry buildings.

From SPO2IDA, three IDA curves are obtained corresponding to the $16 \mathrm{th}, 50 \mathrm{th}$, and 84 th percentiles of a population of IDA curves reflecting the record-to-record variability (e.g. FEMA 1997). The spectral acceleration derived for each limit state $\mathrm{Sa}_{\mathrm{LS}}$ is converted to Peak Ground Acceleration $P G A(g)$ by using a scaling relationship based on a typical ground motion prediction equation (GMPE). The GMPE proposed by Boore et al. (2014) is adopted. The conversion from $\mathrm{Sa}_{\mathrm{LS}}$ to $P G A(g)$ is implemented as shown in Eq. 2:

$P G A(g)=S a_{L S} \cdot \widehat{P G A}_{G M P E}$

where $\widehat{P G A(g)})_{G M P E}$ and $\widehat{\operatorname{Sa}}_{\mathrm{LS}}$ GMPE are the average $P G A(g)$ and the average spectral acceleration obtained using the chosen GMPE, considering (i) the case of normal faulting, (ii) average top-30 m shear wave velocity of $300 \mathrm{~m} / \mathrm{s}$ and (iii) magnitude and distance ranges of $M_{\mathrm{w}} 5$ to $M_{\mathrm{w}} 8$ and 1$30 \mathrm{~km}$, respectively. Once the IDA curves in terms of $P G A$ are available, it is possible to derive the fragility curves.

The approaches adopted for deriving fragility curves for Collapse $(C)$ and Near Collapse (NC) limit states and for Severe Damage $(S D)$ and Light Damage (LD) limit states are different. For the limit states $C$ and $N C$, a fragility curve can be obtained from each SPO curve. This is because the ductility $\left(\frac{\Delta_{c^{*}}}{\Delta_{y}}\right.$ at $C$ and $\frac{\Delta_{u}}{\Delta_{y}}$ at NC) is larger than 1 for $N C$ and $C$, and therefore three IDA curves providing the 16th, 50th, and 84th $P G A(g)$ percentiles can be derived (Fig. 2f). Consequently, for each pushover curve, a fragility curve is obtained according to the following relationship:

$$
\begin{aligned}
P[L S \mid P G A] & =\Phi\left[\frac{\ln (P G A(g))-\ln \left(P G A(g)_{50^{\text {th }}}\right)}{0.5 \cdot \ln \left(P G A(g)_{84^{\text {th }}} / P G A(g)_{16^{\text {th }}}\right)}\right] \\
& =\Phi\left[\frac{\ln (P G A(g))-\ln (\eta)}{\beta}\right]
\end{aligned}
$$

where $\Phi[]$ is the standard normal distribution function and $\eta$ and $\beta$ are the equivalent median and logarithmic standard deviation (i.e. dispersion) of the fragility curves in terms of $P G A(g)$. The final fragility is obtained as the mean fragility of the curves from the analyses of all façades:

$P[L S \mid P G A(g)]=\frac{1}{n} \cdot \sum_{i=1}^{n} P_{i}[L S \mid P G A(g)]$

where $n$ is the number of analysed façades.

For the damage states $S D$ and $L D$, for the ductility is lower or equal to $1\left(\frac{\Delta_{y}}{\Delta_{y}}\right.$ at $S D$ and $\frac{\Delta_{\text {cr }}}{\Delta_{y}}$ at LD). Therefore, the three IDA curves provide a single value of PGA. The three IDA curves corresponding to the 16th, 50th, and 84th $P G A(g)$ percentiles curves coincide for the elastic and quasi-elastic structural behaviour (Fig. 2f). Hence, the final fragility curves are obtained by performing a log-normal regression on the $n P G A(g)$ values obtained for all the façades.

In conclusion, the interpretations of the fragility are different for the nonlinear branches ( $C$ and $\mathrm{NC}$ ) and linear branches ( $S D$ and LD) - for the $C$ and NC cases, record-torecord variability is the main contribution of the fragility, while this uncertainty is suppressed for the $S D$ and $L D$ cases under the assumptions of a linear elastic SDOF oscillator.

\section{Building characterisation}

\subsection{Geometric and structural features of surveyed buildings}

On-site structural surveys were carried out on 323 nonengineered unreinforced masonry buildings located in formal and informal settlements in the townships of Salima and Balaka and informal settlements of the villages Lifidzi and Golomoti (see Fig. 1b). 86\% of the inspected buildings are made of fired (clay) bricks (see Fig. 6a, b), since these materials are relatively cheap to source on-site and do not need specific construction skills. The remaining $14 \%$ of the inspected buildings are made of mud bricks (see Fig. 6c), a 
Typical geometric and structural features of the building typology

(a)

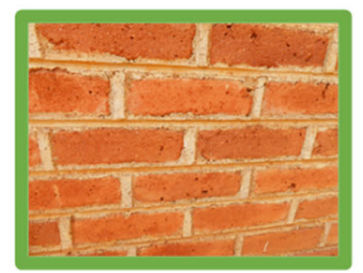

(e)

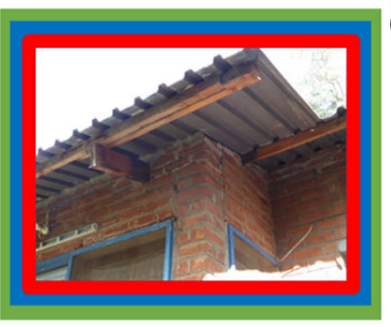

(i)

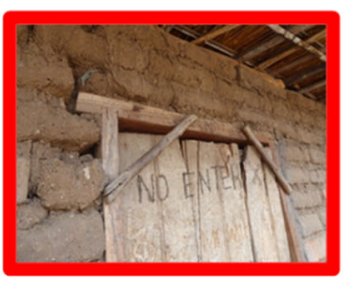

(m)

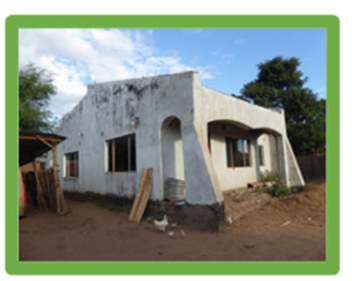

(q)

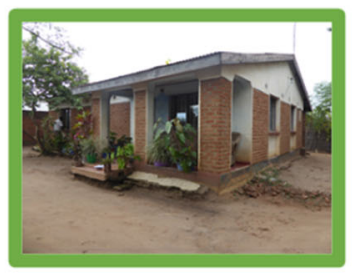

(b)

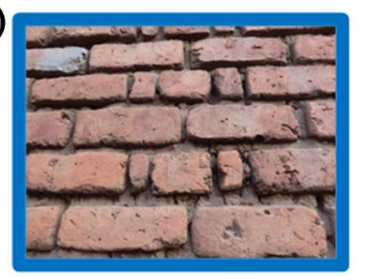

(f)

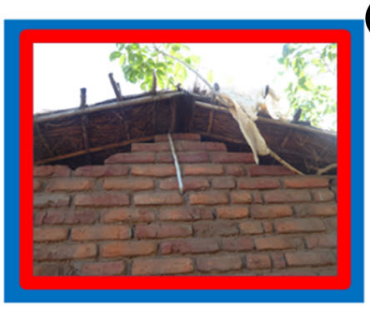

(j)

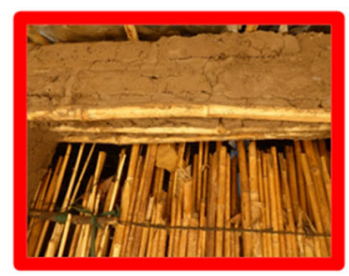

(n)

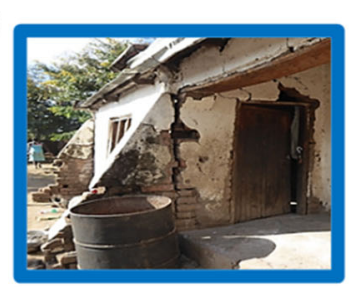

(r)

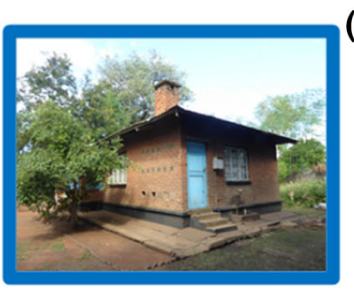

Fig. 6 Typical buildings in Malawi: a fired bricks with cement mortar; b fired bricks with mud mortar; c unfired bricks with mud mortar; $\mathbf{d}$ thatched roof; e light metallic sheets; $\mathbf{f}$ gable detached from the roof structure; $\mathbf{g}$ lack of connection between walls and roof system; $\mathbf{h}$ timber wall plates of circular section; $\mathbf{i}$ door frame showing absence of lintels; $\mathbf{j}$ bamboo lintel; $\mathbf{k}$ concrete lintel; $\mathbf{I}$ house with poor

material commonly used for traditional houses and most of the informal settlements. Mud mortar (Fig. 6b, c) and cement mortar (Fig. 6a) are used for $76 \%$ and $24 \%$ of the inspected buildings, respectively.

The majority of the inspected houses $(60 \%)$ have singleskin walls with thicknesses varying from 100 to $180 \mathrm{~mm}$, while houses with double-skin walls have a thickness varying from 190 to $300 \mathrm{~mm}$. The roofs are made of thatch for $21 \%$ of the inspected buildings, while light metallic

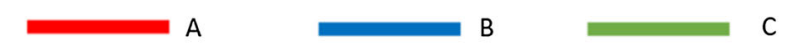

(c)

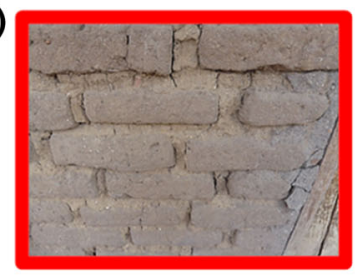

(g)

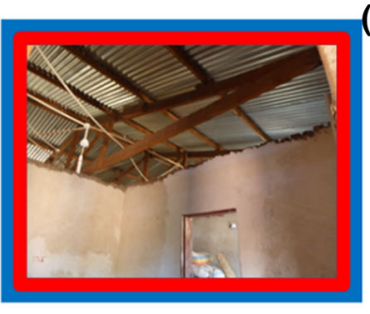

(k)

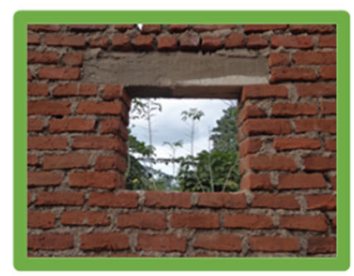

(o)

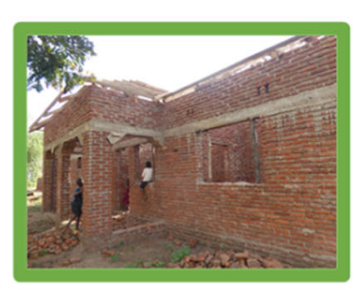

(s)

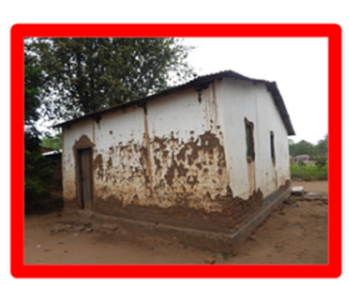

(d)

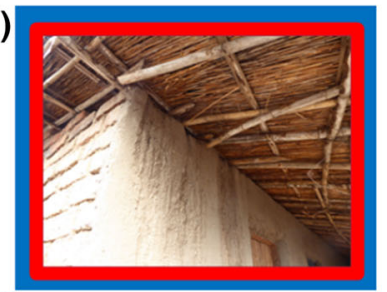

(h)

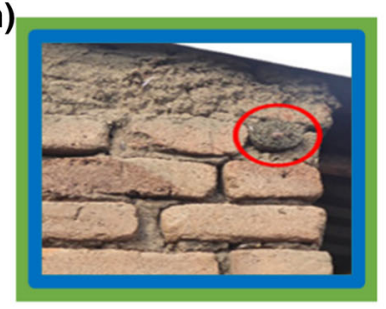

(p)

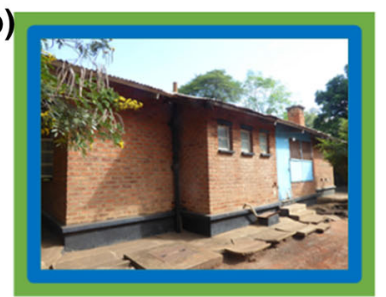

(t)

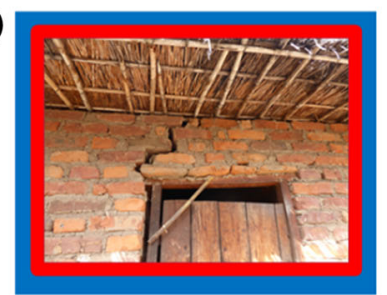

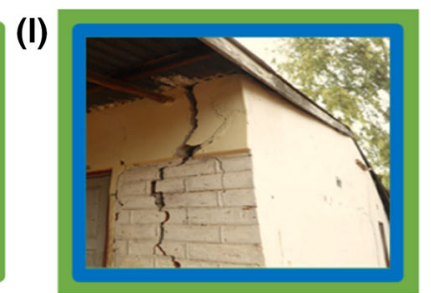

connection between walls; m new constructions with buttresses; $\mathbf{n}$ lack of connection between buttress and wall; $\mathbf{o}$ concrete ring beam; $\mathbf{p}$ re-entrant corner; $\mathbf{q}$ portico; $\mathbf{r}$ chimney; $\mathbf{s}$ material erosion; $\mathbf{t}$ typical cracks. The red-blue-green tag on each picture is used to highlight the geometric/structural features of the building typologies A, B, and C, introduced in Sect. 3.1.1

corrugated sheets are observed for the remaining inspected buildings (Fig. 6e). Both roof types do not act as a rigid diaphragm during an earthquake. Furthermore, roofs generally are not connected to the bearing walls; therefore, they are not able to provide restraint against lateral movements due to winds or earthquakes (Fig. 6f, g). Only occasionally, timber wall plates are used on the top of the longest external walls to support the roof structures 
(Fig. 6h). From the surveys, it also became evident that neither timber ring beams nor tie elements are used.

From a seismic point of view, there are a few positive characteristics of the buildings in the area. All of the surveyed houses are one-storey buildings and have rectangular plans (typical plan dimensions $8 \mathrm{~m} \times 6 \mathrm{~m}$ ). Generally, the longest walls have from 1 to 6 openings. The shortest walls usually have no openings and present gable elements with a height varying from 0.25 to $1.9 \mathrm{~m}$. On the other hand, poor construction detailing significantly compromises the seismic performance of the buildings. Examples of poor detailing are the lack of lintels on top of openings; openings are provided with frames made of timber elements with small cross-sections (typically $10 \mathrm{~cm} \times 5 \mathrm{~cm}$ ) (Fig. 6i). When lintels are adopted, they are made with bamboo elements, according to the traditional practice in the country (Fig. 6j), or, although rarely, with concrete beams (mainly adopted in buildings for wealthy households (Fig. 6k). The poor detailing is also demonstrated by the lack of connections between orthogonal walls (Fig. 6l) and between walls and the roof, which could lead to brittle failures during earthquakes.

For the case of single-skin walls, connections between different elements are generally poor. In contrast, for the double-skin walls, connections between walls and between the walls and roof are often better, and thus provide higher structural stability to the entire building. To improve the quality of connections between walls, a small percentage of the inspected houses $(6 \%)$ have buttresses or ring beams (Fig. 6m-o). In Malawi, buttresses are strengthening elements added to the houses and are also meant to create an additional space (e.g. porch). These elements, when identified in newly constructed residential buildings, are generally made with fired bricks and cement mortar and are well connected to the structure. In contrast, when buttresses are made of fired bricks of poor or medium fabric quality and mud mortar, they are often disconnected from the orthogonal wall to which they are attached, and therefore do not contribute to the stability of the houses and do not prevent Out-of-Plane modes when an earthquake occurs.

Ring beams, which are constructed with reinforced concrete and are only observed in a few new buildings located in the townships, are placed on top of openings, and function as a lintel for distributing loads from the spandrels to the adjacent piers, and as a belt for ensuring connection at the tops of the walls. Seismic performance of the inspected houses could also be vastly affected by the presence of irregular elements (e.g. re-entrant corner, portico, chimney, see Fig. 6m, p, q, r), observed in several new houses. Structural shortcomings in these types of constructions are highly aggravated by lack of maintenance, which inevitably accelerates the deterioration of the main constructional materials and of structural elements in the roof and openings. This is evident from the presence of cracks, material erosion, dry timber rot, damaged boards, and door jams (Fig. 6s, t).

\subsubsection{Building classification}

Table 1 shows the classification of the inspected buildings according to roof type (thatched/metallic sheet), mortar type (mud/cement), fabric quality (1: good, 2 : medium, 3 : poor; see Sect. 2.1), and quality of wall connections (good/ poor). According to the data listed in Table 1, buildings are classified in three typologies: (A) buildings of poor-quality construction; (B) buildings of medium-quality construction and (C) buildings of high-quality construction (see geometric and structural features of the identified typologies in Fig. 6).

Figure 7a shows the total percentages of the building typologies A, B, and C and how these are distributed across the inspected settlements (i.e., Balaka, Golomoti, Lifidzi, and Salima). Figure $7 \mathrm{~b}$ shows the breakdown by typologies. Brief descriptions of the typologies are:

- Typology A-This is representative of $26 \%$ of the inspected buildings, characterised by high seismic vulnerability (Fig. 8a). These buildings are made of

Table 1 Proportions of buildings with reference to main features including fabric quality (1: good; 2 : medium, and 3 : poor) defining the building typologies

\begin{tabular}{|c|c|c|c|c|c|c|c|c|}
\hline \multicolumn{9}{|c|}{ Total number of inspected buildings $=323$} \\
\hline \multirow{2}{*}{\multicolumn{2}{|c|}{$\frac{\text { Roof type }}{\text { Masonry type }}$}} & \multicolumn{7}{|c|}{ Thatched roof } \\
\hline & & \multicolumn{3}{|c|}{ Fired bricks } & & \multicolumn{3}{|c|}{ Unfired bricks } \\
\hline \multicolumn{2}{|l|}{ Mortar type } & \multicolumn{7}{|c|}{ Mud } \\
\hline \multicolumn{2}{|l|}{ Fabric quality } & \multicolumn{2}{|l|}{2} & \multicolumn{2}{|l|}{3} & 1 & 2 & 3 \\
\hline \multicolumn{2}{|c|}{ Poor connection (\%) } & \multicolumn{2}{|l|}{4.3} & \multicolumn{2}{|l|}{2.5} & 1.2 & 11.0 & 1.9 \\
\hline \multirow{2}{*}{\multicolumn{2}{|c|}{ Poor connection $(\%)$}} & \multicolumn{2}{|l|}{0.3} & \multicolumn{2}{|l|}{-} & - & - & - \\
\hline & & \multicolumn{2}{|l|}{ B } & \multicolumn{5}{|l|}{ A } \\
\hline \multirow{2}{*}{$\begin{array}{l}\text { Roof type } \\
\text { Masonry type }\end{array}$} & \multicolumn{8}{|c|}{ Metallic sheet roof } \\
\hline & \multicolumn{2}{|c|}{$\begin{array}{l}\text { Fired } \\
\text { bricks }\end{array}$} & \multicolumn{3}{|c|}{ Unfired bricks } & \multicolumn{3}{|c|}{$\begin{array}{l}\text { Fired } \\
\text { bricks }\end{array}$} \\
\hline Mortar type & \multicolumn{5}{|c|}{ Mud } & \multicolumn{3}{|c|}{ Cement } \\
\hline Fabric quality & 1 & 2 & 3 & 1 & 2 & 3 & 1 & 2 \\
\hline $\begin{array}{l}\text { Poor connection } \\
(\%)\end{array}$ & 1.5 & 20.6 & 6.7 & 0.3 & 2.2 & 0.5 & 0.2 & 2.3 \\
\hline \multirow{2}{*}{$\begin{array}{l}\text { Poor connection } \\
(\%)\end{array}$} & 2.2 & 21.2 & - & - & - & - & 11.5 & 9.6 \\
\hline & B & & A & & & & $\mathrm{C}$ & \\
\hline
\end{tabular}


(a) $60 \%$

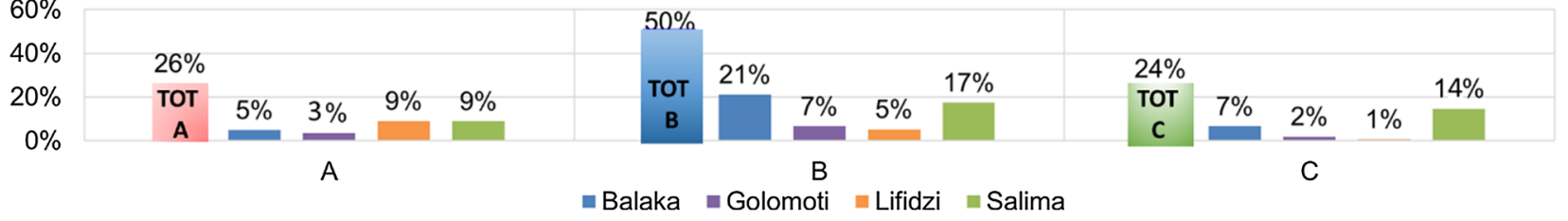

(b) $100 \%$

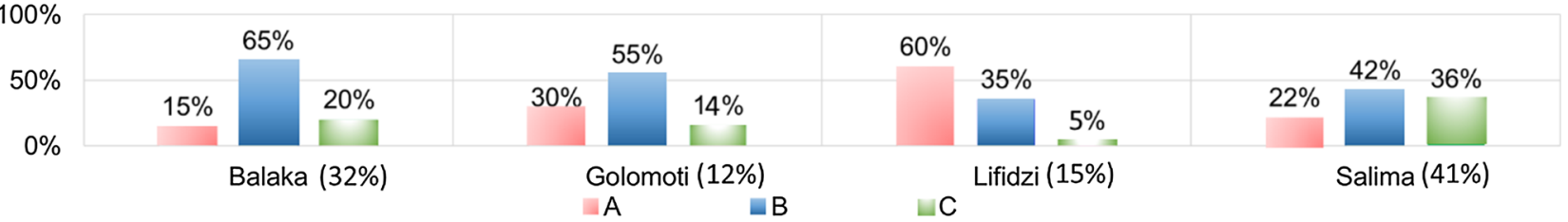

Fig. 7 a Total percentages of building typologies A, B, and C, b percentages of the building typologies A, B and C identified for each inspected settlement

(a)

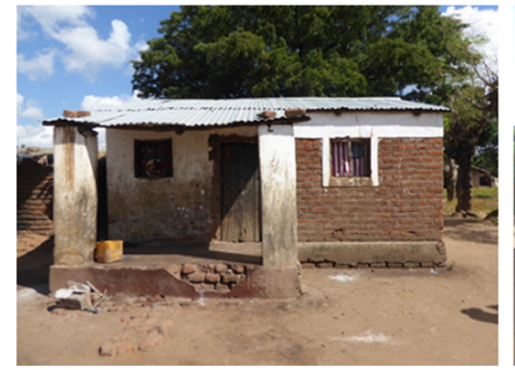

(b)

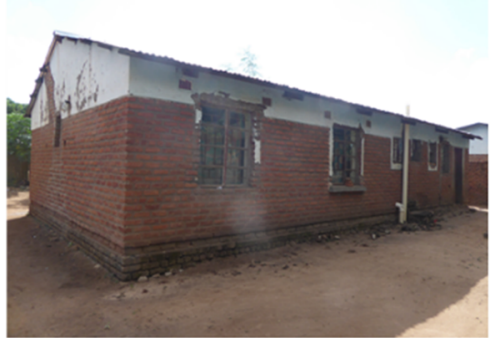

(c)

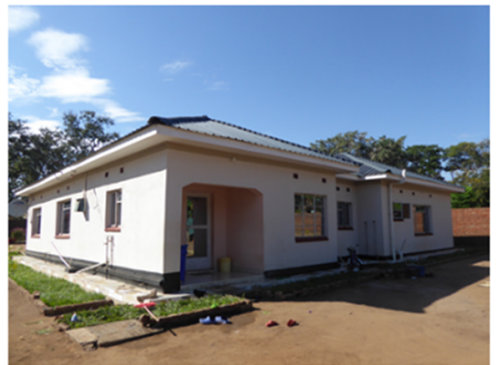

Fig. 8 Typical masonry buildings in Malawi: a typology A; b typology B; c typology C

unfired or fired bricks with mud mortar and are of poor fabric quality (Fig. 3b3). Generally, these houses have smaller building footprints than a typical floor plan of $8 \mathrm{~m} \times 6 \mathrm{~m}$. These houses have thatch or corrugated metallic sheet roofs supported by light timber elements. The structures are characterised by poor structural detailing (e.g. lack of connection between walls and between walls and roof).

- Typology B-This is the most common typology observed in Malawi, representing 50\% of the buildings, and is rated as medium seismic vulnerability (Fig. 8b). These buildings are made of fired bricks with mediumto good-quality fabric (Fig. 3b1, b2). Due to the presence of mud mortar, bonding between bricks is considered poor; therefore, connections between walls are assumed weak. The construction details varied significantly as well as maintenance levels.

- Typology C-This covers $24 \%$ of the inspected houses and is rated as low seismic vulnerability (Fig. 8c). These were made of fired bricks with medium- to goodquality fabric (Fig. 3b1, b2) and cement mortar. Generally, these houses have a larger floor plan than a typical plan. Due to the extended plan size, irregularities are likely to occur (e.g. portico and re-entrant corner). Most of these houses have corrugated metallic sheets supported by timber elements or trusses, and good structural detailing (e.g. adjacent walls and walls/ roof are well connected). The good structural quality of these houses can also be attributed to the presence of strengthening elements (e.g. ring beams).

\subsubsection{Expected failure modes}

Possible failure modes for buildings in Malawi are identified based on the data from the post-earthquake surveys carried out after the 2009 Karonga earthquake sequence by the authors. Four failure modes are identified, as shown in Fig. 9:

(a) GABLE failure: according to post-earthquake field observations, this failure predominantly occurs on building typologies A and B in walls with gables which are not connected to roofs, and therefore fail in overturning. This failure mode is characterised by an inverted arch crack pattern on the gable wall.

(b) OOP (Out-of-Plane of the entire façade) failure: according to post-earthquake field observations, this failure mainly occurs on building typologies A and B made of single-skin walls with poor-quality materials and poor connections between walls and between walls and the roof, causing overturning of a single 


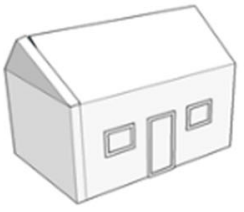

(a) GABLE

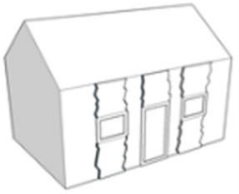

(c) STRIP

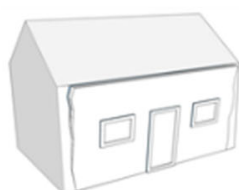

(b) OOP (a)

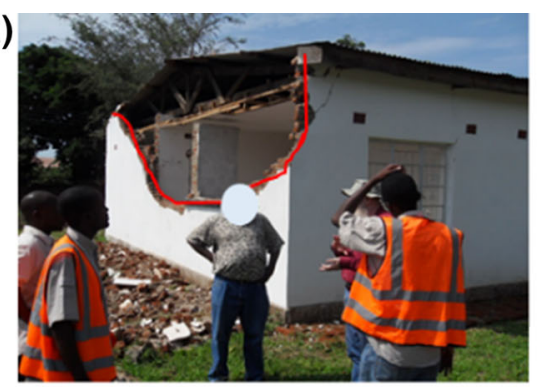

(c)

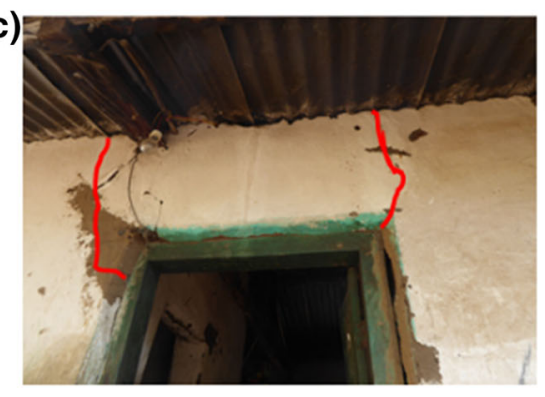

(b)

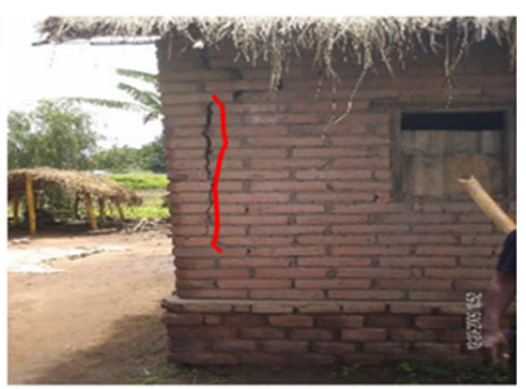

(d)

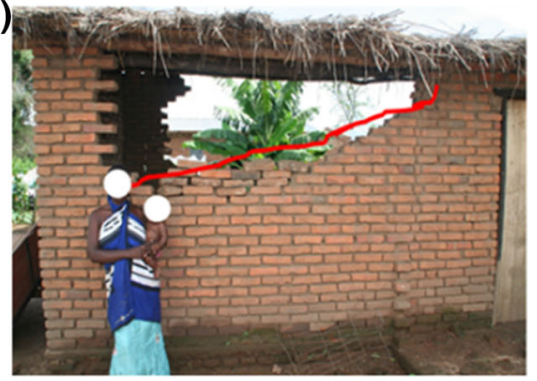

Fig. 9 Typical failure modes observed after the 2009 Karonga earthquake sequence. a OOP, b GABLE, c STRIP, d IP. Source of image for ac are taken from the authors $\mathbf{d}$ (http://www.aaronmoore.com.au/malawi-earthquake-relief\#/i/1) taken by Aaron Moore, used with permission

façade. This failure mode is characterised by vertical cracks on the edges of the wall.

(c) STRIP failure: according to post-earthquake field observations, this failure predominantly occurs on building typologies $\mathrm{B}$ and $\mathrm{C}$ made of single-/doubleskin walls with medium-quality materials and good connections between walls and between walls and the roof, causing overturning of vertical strips of piers or spandrels. This failure mode is characterised by vertical cracks along the edges of piers or spandrels aligned vertically.

(d) IP (In-Plane) failure: according to post-earthquake field observations, this failure predominantly occurs on building typology $\mathrm{B}$ and $\mathrm{C}$ made of double-skin walls with medium- to good-quality materials and good-quality connections between walls and between walls and the roof, causing shear failure of a single façade. This failure mode is characterised by a diagonal crack on the entire wall or diagonal or $\mathrm{X}$-shape cracks on piers or spandrels. Buildings built according to the Safer House Construction Guidelines (Bureau TNM 2016) are likely to fail in this mode.

\subsection{Experimental campaign}

The results from the tests (Sect. 2.2) indicate that the behaviour of the masonry in compression is governed by the low compressive strength of the bricks. The quality of the brick-mortar bonding governs the In-Plane shear and
Out-of-Plane flexural behaviour, which are the critical parameters of the resistance to horizontal loadings, such as earthquake action.

To be consistent with the poor brick-mortar bonding measured in the tested couplets and triplets made with bricks and mud mortar, the connections between single-/double-skin walls for buildings in typology $\mathrm{A}$ and typology B (made of fired and undried bricks, respectively, and mud mortar) are assumed insufficient. Conversely, for typology $\mathrm{C}$ made of single-/double-skin walls with fired bricks and cement mortar, it is assumed that masonry walls are well connected.

To characterise the different level of material deterioration, the fired bricks with good fabric quality for buildings in typology $\mathrm{C}$ are assumed to be soaked in water prior to construction. Therefore, for these buildings the cementto-sand ratio of $1: 6 \mathrm{~F}$ (in agreement with the recommendations of the Guidelines) is considered, while for all other buildings in typology $\mathrm{C}$ with medium and low fabric quality, the cement-to-sand ratio of 1:8U (most affordable and adopted by local artisans) is deemed suitable (see Sect. 2.2 for the definitions of $F$ and $U$ ).

To characterise brick-mortar bonding of the typologies $\mathrm{A}, \mathrm{B}$, and $\mathrm{C}$, the interface cohesion and friction angle are defined with reference to the values measured during the tests. The adopted values are:

- for buildings with mud mortar in typologies A and B, the interface cohesion varies from $0.01 \mathrm{MPa}$ (poor fabric quality of masonry) to $0.02 \mathrm{MPa}$ (good fabric quality of masonry). These are the minimum and 
maximum values found for the tests on mud mortar (Kloukinas et al. 2019).

- for buildings with the strongest cement mortar (1:6F) in typology $\mathrm{C}$, the interface varies from $0.06 \mathrm{MPa}$ (poor fabric quality masonry) to $0.12 \mathrm{MPa}$ (good fabric quality masonry). These are the minimum and maximum values found for the tests on cement mortar (1:6F) (Kloukinas et al. 2019).

- for buildings with the poorest cement mortar (1:8U) in typology $\mathrm{C}$, the interface varies from $0.04 \mathrm{MPa}$ (poor fabric quality masonry) to $0.08 \mathrm{MPa}$ (good fabric quality masonry). These are the minimum and maximum values found for the tests on cement mortar (1:8U) (Kloukinas et al. 2019).

- for all three typologies, friction angles are about 32 degrees (Kloukinas et al. 2019).

\section{Seismic building performance}

\subsection{Failure mode analysis of the inspected buildings}

The results shown in this section are derived from the analyses of all the inspected façades using FaMIVE (D'Ayala and Speraza 2003), introduced in Sect. 2.3. As expected from the on-site surveys, due to a lack of connections between the walls and roof and frequent use of poor-quality construction materials, OOP is the most likely failure mode with a percentage of $42 \%$, followed by GABLE (25\%), STRIP (25\%), and IP (8\%), see failure mode proportions in Fig. 10a. Similar failure mode proportions are also observed for each individual settlement. Since OOP, GABLE, and STRIP are more likely to happen, buildings in Malawi would not exhibit a box behaviour under seismic loadings. The failure mode proportions for building typologies underline that buildings of typology A, with low construction quality, have the highest percentage of constructions failing in OOP; this is 56\%, against $13 \%$ estimated for buildings in typology $\mathrm{C}$, characterised by high construction quality. It is also interesting to observe that GABLE failures cover an important percentage in all building typologies, and is high in typology $\mathrm{C}$, underlining that the current practice needs to improve connectivity between masonry walls, wall plates, which are generally absent, and gable panels. For typology B, characterised by houses of medium construction quality, failure mode proportions show that overturning of gables, walls, spandrels, and piers as wells as In-Plane failure of walls are typical failure modes for this typology.

The cumulative damage curves of Fig. 10b-d plot the values of the collapse load factor multiplier for each analysed building against the number and percentage (on the left and right of each plot, respectively) of collapsed buildings. They provide the average of $\lambda$ for the inspected locations, failure modes, and building typologies. In Fig. 10b, the slopes and relative positions of the curves underline that the rural settlements Golomoti and Lifidzi have weaker constructions than the urban settlements Balaka and Salima. These results are expected considering that Golomoti and Lifidzi have the highest percentages of buildings in typology A $(30 \%$ and $60 \%$, respectively, as illustrated in Fig. 7b), while Balaka and Salima have the highest percentages of buildings in typology $\mathrm{C}(20 \%$ and $36 \%$, respectively, as illustrated in Fig. 7b). Figure 10c shows that GABLE and OOP are the most critical failure modes for most of the inspected buildings as highlighted by the failure mode percentages reported for $\lambda=0.30$ each failure mode class. The results are also confirmed by the average values of $\lambda$ which are higher for STRIP and IP than the others, since these failure modes mainly occur for buildings in typologies $\mathrm{B}$ and $\mathrm{C}$ of medium or high construction quality, as indicated in the failure mode proportions of Fig. 10a. As expected, the slopes of the cumulative damage curves in Fig. 10d highlight that building typology A, failing mainly in GABLE and OOP as illustrated in Fig. 10a, is the most vulnerable to seismic loading, followed by typologies $\mathrm{B}$ and $\mathrm{C}$. This is consistent with the average values of $\lambda$ (i.e. $\lambda=0.19,0.33$, and 0.41 for $\mathrm{A}, \mathrm{B}$, and $\mathrm{C}$, respectively) and the percentages of collapsed buildings for $\lambda=0.30$.

\subsection{Derivation of SPO curves for equivalent SDOF systems}

The effects of the behavioural uncertainty due to (1) geometric instability, (2) limited ductility, and (3) degradation of strength on the SPO curves are investigated. In Fig. 11, results based on the three models show the SPO curves obtained for each inspected façade in comparison with their related average calculated on the entire inspected building stock. Furthermore, in Fig. 11a, b the SPO curves are expressed as an average of the capacity curves calculated on subsets of the inspected façades failing in GABLE, OOP, STRIP, and IP, and classified in typologies A, B, and $\mathrm{C}$, respectively.

The uncertainty regarding the three models of structural behaviour is highlighted in Fig. 11 by the three different sets of backbone SPO shapes. These differ in strength and ductility, demonstrating the impact of different definitions of the limit states described in Sect. 2.5 for each model. The highest strength is observed in the façades with limited ductility, as they have an average spectral acceleration $\left(S a_{\max }\right)$ which is $22 \%$ greater than the ones obtained in the 
(a)

\begin{tabular}{|c|c|c|c|c|c|}
\hline & GABLE & OOP & STRIP & IP \\
\hline & TOTAL & $25 \%$ & $42 \%$ & $25 \%$ & $8 \%$ \\
\hline Balaka (32\%) & $18 \%$ & $30 \%$ & $35 \%$ & $17 \%$ \\
\hline Salima (41\%) & $26 \%$ & $41 \%$ & $29 \%$ & $4 \%$ \\
\hline Golomoti (12\%) & $19 \%$ & $51 \%$ & $21 \%$ & $9 \%$ \\
\hline Lifidzi (15\%) & $38 \%$ & $57 \%$ & $5 \%$ & -- \\
\hline A (26\%) & $27 \%$ & $56 \%$ & $15 \%$ & $2 \%$ \\
\hline B (50\%) & $20 \%$ & $49 \%$ & $16 \%$ & $14 \%$ \\
\hline C (24\%) & $34 \%$ & $13 \%$ & $53 \%$ & -- \\
\hline
\end{tabular}

(c) 120

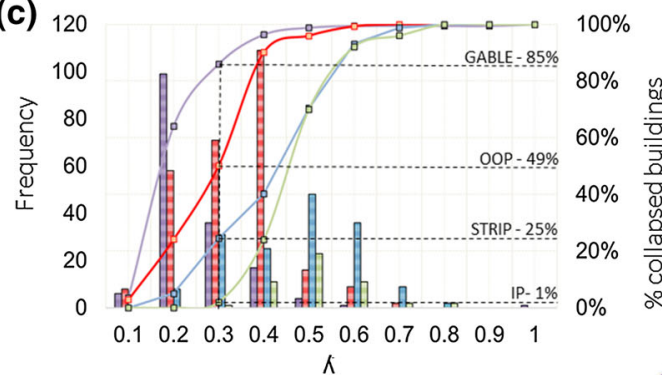

\begin{tabular}{|c|c|c|c|}
\hline \multicolumn{2}{|c|}{$\begin{array}{l}\square \text { GABLE Frequency } \\
\square \text { STRIP Frequency } \\
\propto \text { GABLE } \\
\text {-STRIP }\end{array}$} & \multicolumn{2}{|c|}{$\begin{array}{l}\text { ĐOOP Frequency } \\
\square \text { IP Frequency } \\
- \text { OOP } \\
- \text { IP }\end{array}$} \\
\hline GABLE & OOP & STRIP & IP \\
\hline 0.17 & 0.26 & 0.43 & 0.45 \\
\hline
\end{tabular}

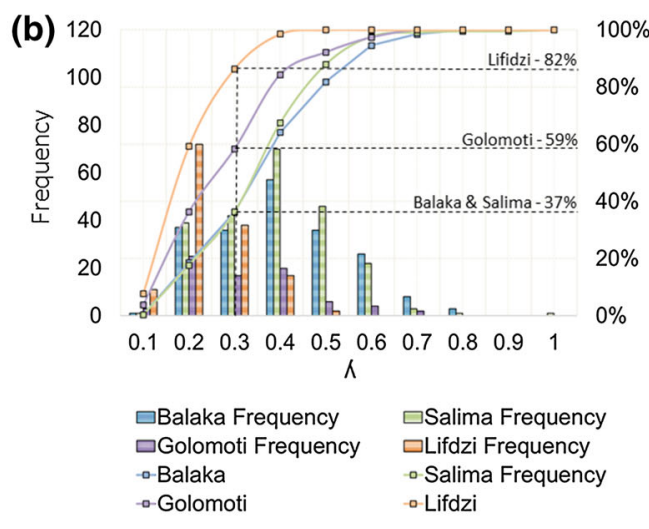

\begin{tabular}{|c|c|c|c|}
\hline Balaka & Salima & Golomoti & Lifidzi \\
\hline 0.30 & 0.34 & 0.26 & 0.19 \\
\hline \multicolumn{3}{|c|}{ Average $\Lambda$} \\
\hline
\end{tabular}

(d)

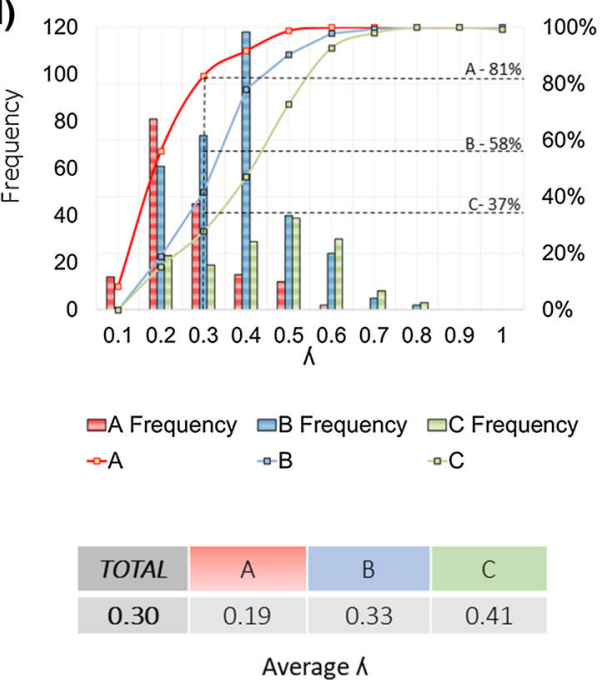

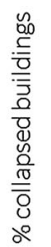
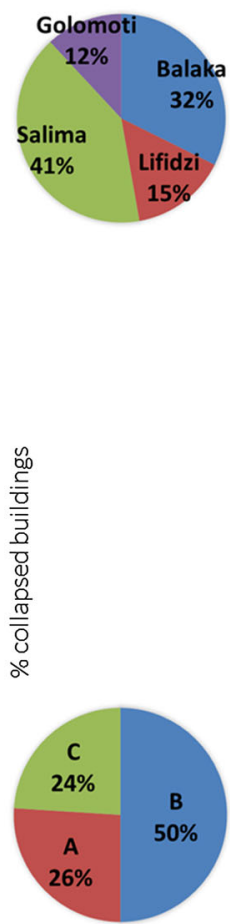

Fig. 10 a Proportions of buildings failing in each mode for the entire inspected building stock, in terms of overall total, locations and building typologies. Cumulative damage curves for $\mathbf{b}$ locations; $\mathbf{c}$ failure modes; $\mathbf{d}$ building typologies

other two models. The median ductility $\left(\mu=\frac{\Delta_{u}}{\Delta_{v}}\right)$ in the façades with degradation of strength results is $19 \%$ lower than the one obtained for façades with geometric instability, while the ductility is 1 for the façades with limited ductility by definition, as described in Sect. 2.5.

Comparing the averages of the SPO curves obtained for different failure modes and building typologies, it is notable that the weakest constructions are the ones failing in GABLE and OOP in Fig. 11a, and the ones classified as building typology $\mathrm{A}$ in Fig. 11b. This is highlighted by their values of $\mathrm{Sa}_{\max }$, which are the lowest compared to the ones estimated for the other classes. This is an expected result, taking into account that $\mathrm{Sa}_{\max }$ is defined as a function of the collapse load factor multiplier $\lambda$ (Sect. 2.4). Note that the average values of $\lambda$ for the weakest classes are lower than the ones estimated for failure modes STRIP and IP and building typologies B and C (Sect. 4.1).
Furthermore, it can be observed that façades failing in IP, which are the ones with better construction quality, have the highest stiffness and the lowest ductility $\mu$. Their SPO backbone curves depict brittle shear failure, which is related to their rigid construction. This is indicated by the value of their average fundamental period $\left(T_{\text {eq }}\right)$, which is equal to $0.04 \mathrm{~s}$ (the shortest among the four), compared to $T_{\text {eq }}$ of $0.38 \mathrm{~s}, 0.40 \mathrm{~s}$, and $0.32 \mathrm{~s}$ calculated for GABLE, OOP, and STRIP, respectively. Façades failing in IP are only a small percentage (7\%) of the total inspected façades (Sect. 4.1) and they do not have a significant influence on the average SPO curves derived for the building typologies in Fig. 11b. This is also confirmed by the similar average fundamental periods $\left(T_{\text {eq }}\right)$ identified for the different building typologies: $0.34 \mathrm{~s}$ for $\mathrm{A}$ and $0.35 \mathrm{~s}$ for $\mathrm{B}$ and $\mathrm{C}$. 


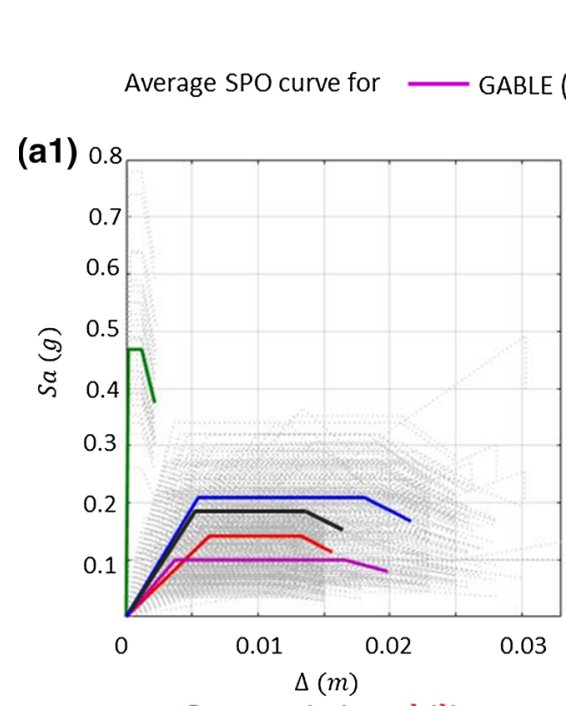

Geometric instability
........... SPO curve for façade $(\mathrm{n}=646)$

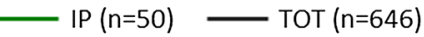

(a2)

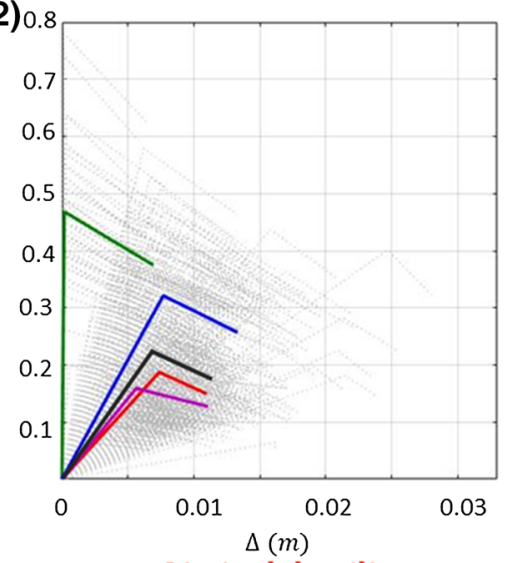

Limited ductility (a3)

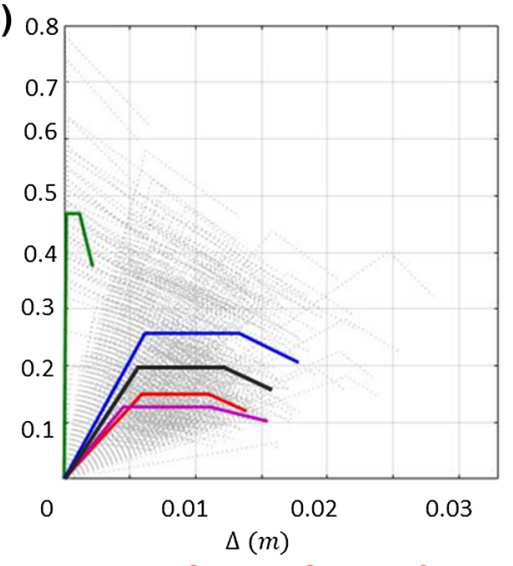

Degradation of strength

........... SPO curve for façade $(n=646)$

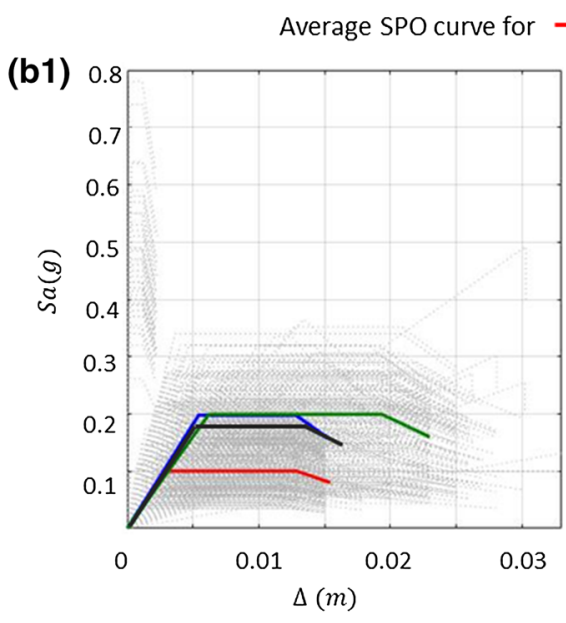

Geometric instability

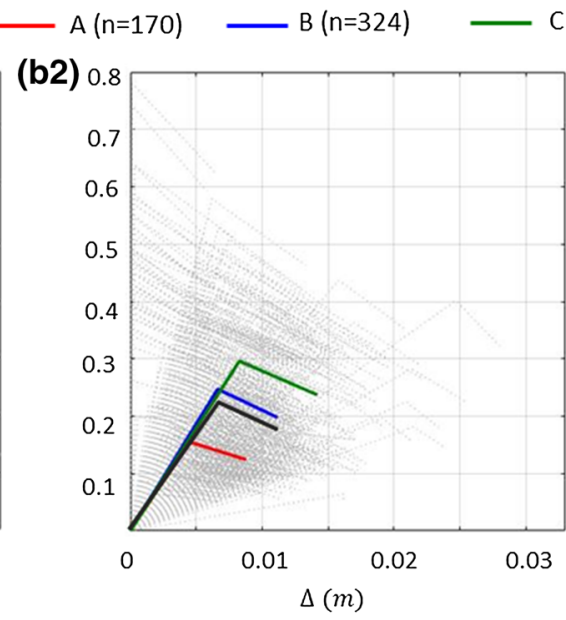

Limited ductility
$C(n=152) \quad \longrightarrow$ TOT $(n=646)$

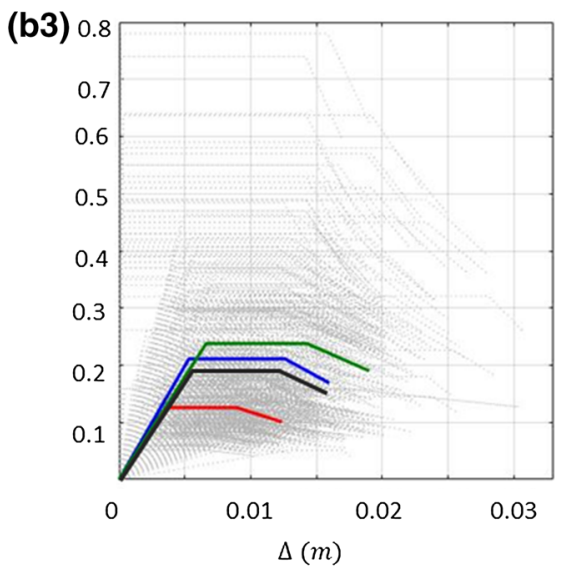

Degradation of strength
Fig. 11 SPO curves for each façade and average SPO curve over all inspected façades, a average SPO curves for failure modes: GABLE, OOP, STRIP and IP, $\mathbf{b}$ average SPO curves for building typologies A,

\subsection{Fragility functions for non-engineered masonry buildings in Malawi}

In order to predict the number of buildings exceeding the limit states defined in Sect. 2.5, fragility curves are developed for failure mode types (Sect. 4.3.1) and building typologies (Sect. 4.3.2), vulnerability classes defined in Sect. 3.1.1 and 3.1.2 according to criteria which describe the order of importance in the parameters (e.g. material types, quality of constructions, level of connection between walls, and presence of gables) affecting the seismic vulnerability of non-engineered houses. The fragility curves are also defined (Sect. 4.3.3) for a weighted model which reflects the real percentage of buildings defined for each
B, C, and total, for (1) geometric instability, (2) limited ductility, and (3) degradation of strength, respectively

identified vulnerability class. Using $P G A(g)$ as the input hazard parameter, three sets of fragility curves are derived, one for each behavioural model introduced in Sect. 2.5, to describe the seismic performance and uncertainty associated with geometric instability, limited ductility, and degradation of strength of the analysed buildings. Since the fragility curves from the different behavioural models have similar shapes, in Figs. 12 and 13 the fragility curves are only presented for geometric instability, whereas the fragility curves for the other structural behaviours are presented in "Appendix". However, the results are discussed illustrating the behavioural uncertainty derived from the three sets of fragility curves listed in Table 2 and later tables, where the probability of exceeding each LS for 
Failure mode classes - Proposed fragility curves for limit states

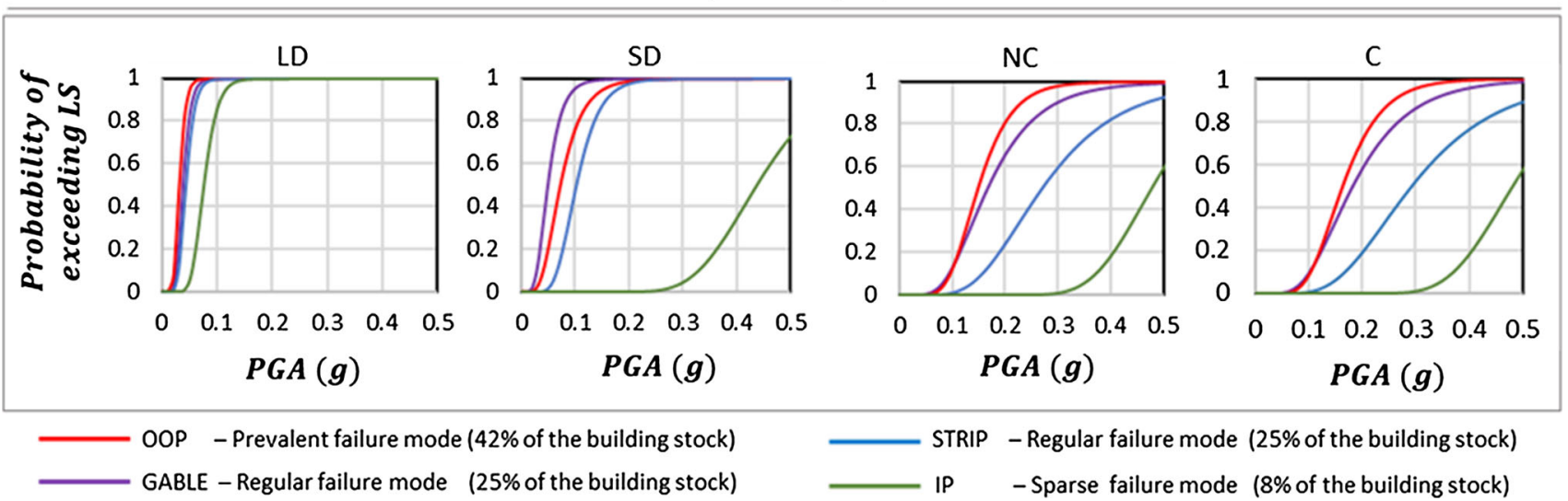

Fig. 12 Failure mode classes. Fragility curves for geometric instability

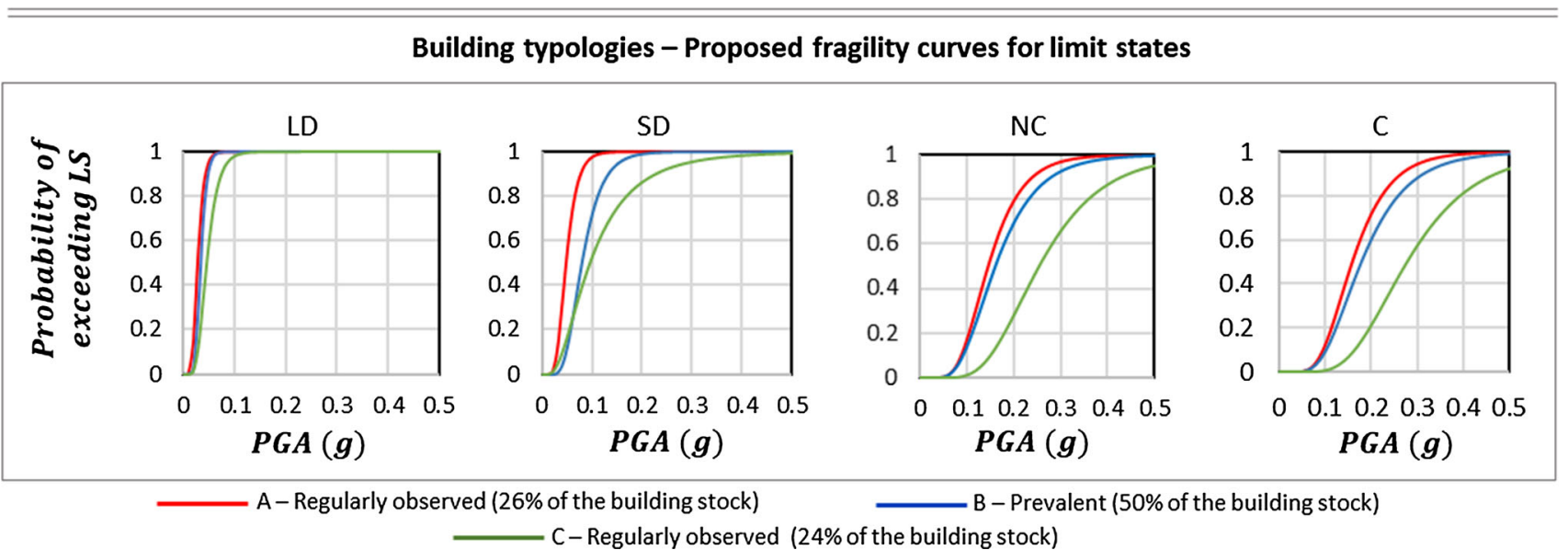

Fig. 13 Building typologies. Proposed fragility curves for geometric instability

different values of $P G A(g)$ are reported as ranges bounded by two values, i.e. the minimum and maximum values from the three behavioural models.

After a discussion on the proposed fragility curves, these fragility functions for building typologies and the weighted model are compared with previously available international reference data/models in Figs. 14 and 15. Note the comparison is only in terms of building typologies (not for failure mode types) as these are the only vulnerability classes provided by the international databases.

\subsubsection{Fragility functions for failure mode classes}

Firstly, the fragility curves are distinguished for the failure mode types (i.e. GABLE, OOP, STRIP, and IP) identified as a critical failure mode of the surveyed non-engineered masonry houses by the mechanical analysis discussed in Sect. 4.1. These fragility curves reported in Fig. 12, are obtained using the fragility parameters (i.e. $\eta$ and $\beta$ ) of
Table 5a. As expected, the fragility curves show that houses failing for overturning of gables, entire façades and vertically alignment of piers or spandrels (i.e. GABLE, OOP, and STRIP) are the most vulnerable even for earthquakes of low intensity.

This is also pointed out in Table 2, where the probability of collapse for large $P G A(g)$ values (i.e. 0.3 and 0.4$)$ is between 0 and $18 \%$ for IP, whereas this probability becomes much higher, up to $99 \%$, for the overturning failure modes (e.g. for $P G A(g)=0.4$ the probability of collapse for OOP is 98-99\%). According to the postearthquake surveys carried out by the authors after the Karonga earthquake in 2009, houses failing in IP are the ones built according to the recommendations provided by the Safer House Construction Guidelines (Bureau TNM 2016). These houses are made of double-skin walls with fired bricks and cement mortar and according to the structural surveys they only represent $8 \%$ of the inspected buildings (see Sect. 4.1). This underlines that although 
Table 2 Failure mode classes

\begin{tabular}{llllll}
\hline Class & LS & \multicolumn{4}{l}{ Probability of exceeding LS at PGA $(\mathrm{g})$} \\
\cline { 3 - 6 } & & $0.1(\%)$ & $0.2(\%)$ & $0.3(\%)$ & $0.4(\%)$ \\
\hline OOP & LD & 100 & 100 & 100 & 100 \\
& SD & $52-77$ & 100 & 100 & 100 \\
& NC & $10-11$ & $81-87$ & $99-100$ & 100 \\
& C & $6-13$ & $71-87$ & $94-97$ & $98-99$ \\
GABLE & LD & 100 & $73-95$ & 100 & 100 \\
& SD & $73-96$ & 100 & 100 & 100 \\
& NC & $11-12$ & $66-80$ & $90-99$ & 100 \\
& C & $9-19$ & $58-79$ & $86-96$ & $96-99$ \\
STRIP & LD & 100 & 100 & 100 & 100 \\
& SD & $7-35$ & $78-100$ & 100 & 100 \\
& NC & 0 & $24-27$ & $60-67$ & $82-89$ \\
& C & $1-2$ & $20-27$ & $52-69$ & $78-92$ \\
& LD & 87 & 100 & 100 & 100 \\
& SD & 0 & 0 & 4 & 34 \\
& NC & 0 & 0 & 0 & 18 \\
& C & 0 & 0 & $0-2$ & $13-18$ \\
\hline
\end{tabular}

Probability of exceeding the limit states for the proposed classification existing guidelines provide appropriate recommendations to enhance the building performance, there is still a low proportion of the existing houses constructed to be safer to earthquakes.

\subsubsection{Fragility functions for building typologies}

Secondly, the fragility curves are distinguished by the building typologies (i.e. A, B, and C) identified as representative vulnerability classes of the surveyed non-engineered masonry houses according to construction materials, roof type and quality of constructions, defined in Sect. 3.1. The building classification uses parameters which are typically adopted in international databases to classify building in terms of seismic vulnerability. Fragility curves reported in Fig. 13 are obtained using the fragility parameters (i.e. $\eta$ and $\beta$ ) reported in Table 6a. As expected, the fragility curves show that poor (A) and medium (B) construction quality buildings (which are also the ones mostly failing in overturning failure modes) are the most vulnerable compared to high (C) construction quality buildings, even for earthquakes of low shaking intensity. This is also evident in Table 3, where the probability of collapse for buildings in typologies A and B are already

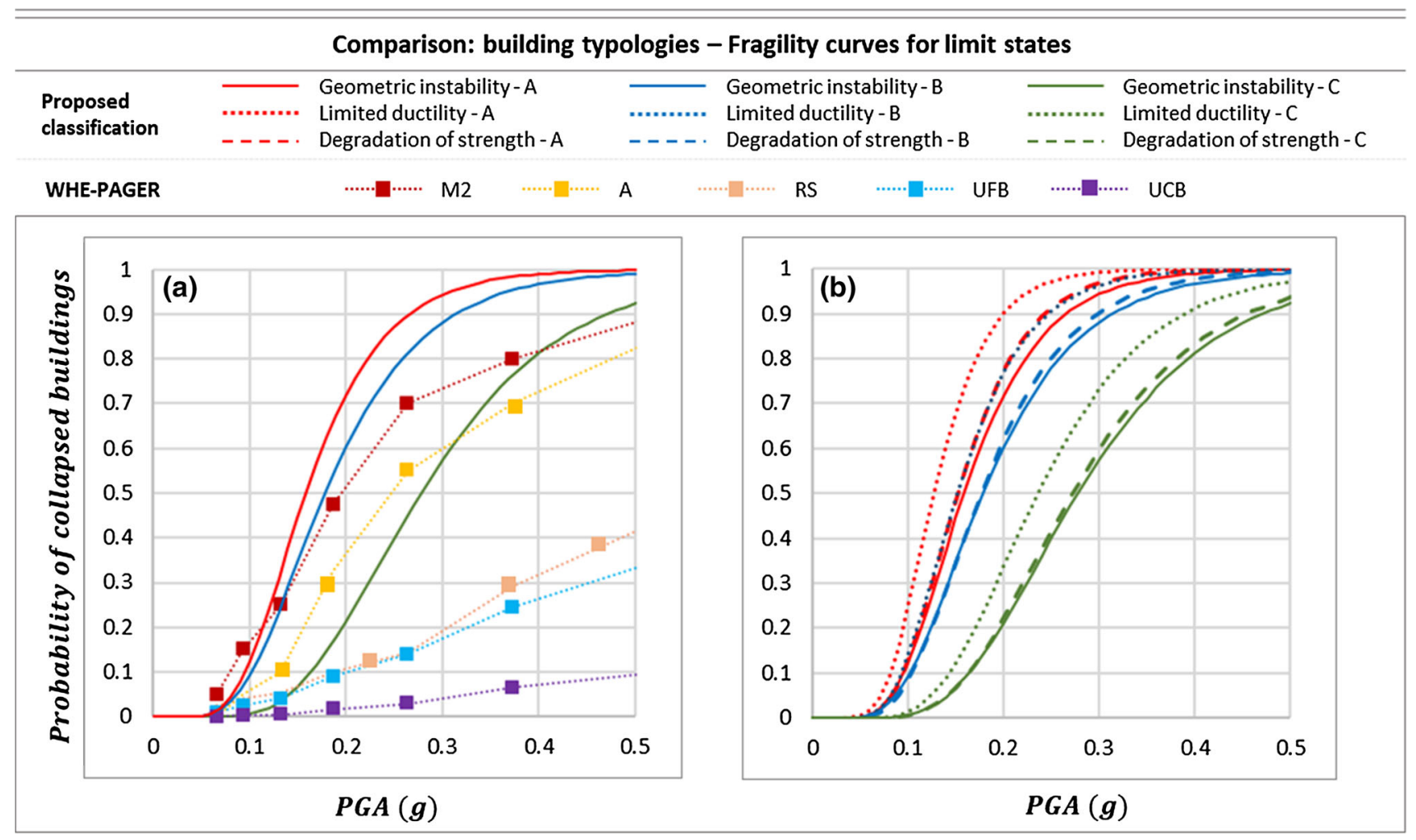

Fig. 14 Comparison: building typologies, a proposed fragility curves for geometric instability and fragility curves by WHE-PAGER classifications, b proposed fragility for geometric instability, limited ductility, and degradation of strength. The comparison shows only the probability of collapsed buildings as this is the only limit state provided by the WHE-PAGER classifications 
Comparison: weighted model - Fragility curves for limit states

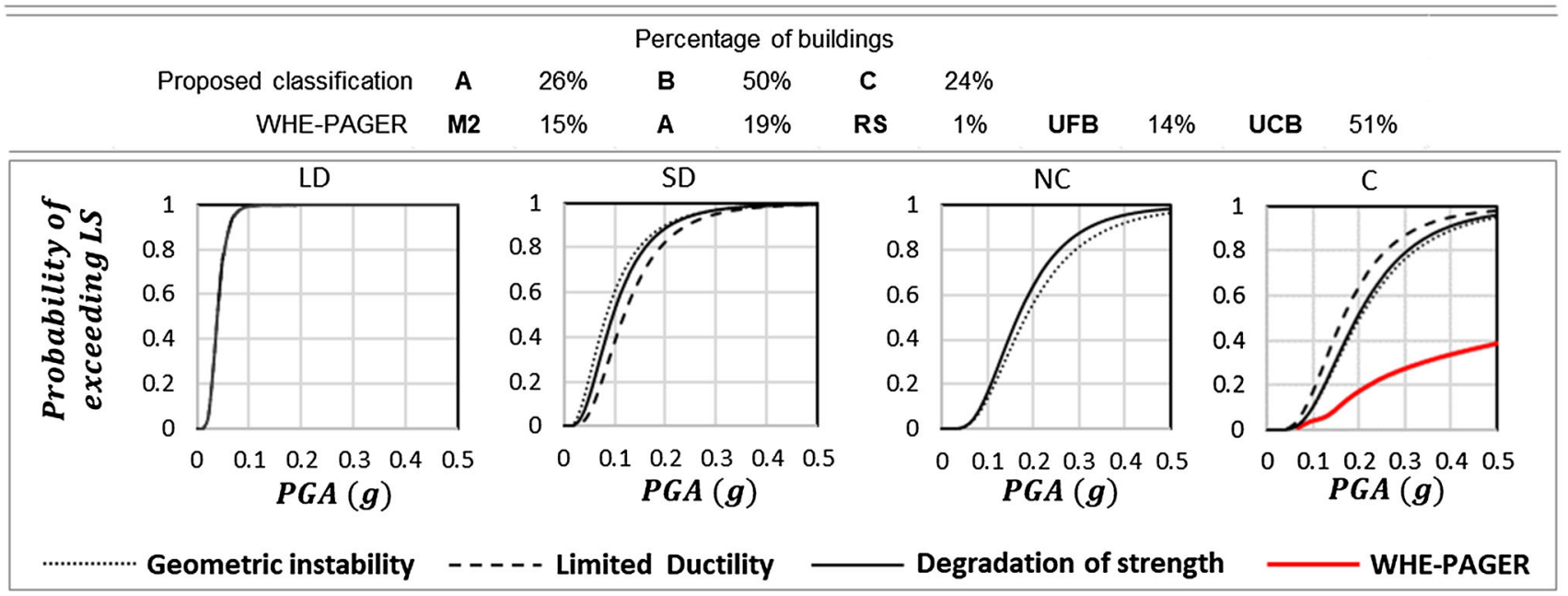

Fig. 15 Comparison: weighted model. Proposed fragility curves for geometric instability, limited ductility, and degradation of strength and fragility curves by WHE-PAGER classifications. Only the probability of collapsed buildings is shown for WHE-PAGER as this is the only limit state provided by this classification

Table 3 Comparison: building typologies

\begin{tabular}{|c|c|c|c|c|c|c|c|c|}
\hline & \multirow[t]{2}{*}{ Class } & \multirow[t]{2}{*}{$\mathrm{LS}$} & \multirow{2}{*}{$\begin{array}{l}\text { PAGER. Most } \\
\text { likely vul.class }\end{array}$} & \multirow[t]{2}{*}{ Proposed classification } & \multicolumn{4}{|c|}{ Probability of collapse at PGA (g) } \\
\hline & & & & & $0.1(\%)$ & $0.2(\%)$ & $0.3(\%)$ & $0.4(\%)$ \\
\hline \multirow[t]{12}{*}{ Proposed classification } & \multirow[t]{4}{*}{ A } & $\mathrm{LD}$ & \multirow[t]{4}{*}{ M2 } & \multirow[t]{4}{*}{-} & 100 & 100 & 100 & 100 \\
\hline & & $\mathrm{SD}$ & & & $80-97$ & 100 & 100 & 100 \\
\hline & & $\mathrm{NC}$ & & & $19-20$ & $75-90$ & $98-100$ & 100 \\
\hline & & $\mathrm{C}$ & & & $5-11$ & $68-90$ & $95-100$ & 100 \\
\hline & \multirow[t]{4}{*}{ B } & LD & \multirow[t]{4}{*}{ UFB } & \multirow[t]{4}{*}{-} & 100 & 100 & 100 & 100 \\
\hline & & $\mathrm{SD}$ & & & $40-90$ & $97-100$ & 100 & $! 00$ \\
\hline & & $\mathrm{NC}$ & & & $12-13$ & $69-75$ & $92-96$ & $98-100$ \\
\hline & & $\mathrm{C}$ & & & $2-10$ & $60-77$ & $88-96$ & $96-100$ \\
\hline & \multirow[t]{4}{*}{$\mathrm{C}$} & $\mathrm{LD}$ & \multirow[t]{4}{*}{ UFB } & \multirow[t]{4}{*}{-} & 98 & 100 & 100 & 100 \\
\hline & & $\mathrm{SD}$ & & & $25-53$ & $73-85$ & $91-96$ & 97-98 \\
\hline & & $\mathrm{NC}$ & & & 1 & $27-34$ & $65-73$ & $86-91$ \\
\hline & & $\mathrm{C}$ & & & 0 & $21-33$ & $57-73$ & $81-91$ \\
\hline \multirow[t]{5}{*}{ WHE-PAGER } & M2 & $\mathrm{C}$ & - & $\mathrm{A}$ & 23 & 66 & 77 & 88 \\
\hline & A & $\mathrm{C}$ & - & - & 9 & 51 & 65 & 82 \\
\hline & RS & $\mathrm{C}$ & - & - & 5 & 14 & 24 & 41 \\
\hline & UFB & $\mathrm{C}$ & - & $\mathrm{B}$ and $\mathrm{C}$ & 4 & 13 & 21 & 33 \\
\hline & $\mathrm{UCB}$ & $\mathrm{C}$ & - & - & 0 & 3 & 5 & 9 \\
\hline
\end{tabular}

Probability of exceeding the limit states by the proposed classification and WHE-PAGER classifications. Only the probability of collapsed buildings is shown for WHE-PAGER as this is the only limit state provided by this classification 
extremely high for earthquakes with a $P G A(g)$ of 0.2 against buildings in typologies $\mathrm{C}$ which collapse for earthquakes with a $P G A(g)$ of 0.3 .

In Fig. 14a, the proposed fragility curves for A, B, and C are compared with the building typologies defined by the international building classification scheme proposed by Jaiswal et al. (2011) for Malawi. The Jaiswal et al.'s model is based on the WHE-PAGER classification system (Jaiswal and Wald, 2008), where residential buildings are categorised by classifying country specific buildings by construction materials (Goda et al. 2016). Using the building survey results and demographic information available from the 2008 Malawi census, this model considers five different building typologies for Malawi, and these are mud walls (M2), adobe blocks (A), rubble stone (RS), unreinforced fired brick masonry (UFB), and concrete block unreinforced masonry (UCB). For making a complete comparison and highlighting the behavioural uncertainty from a graphical point of view, the proposed fragility curves for building typologies are presented not only for geometric instability but also for limited ductility and degradation of strength (Fig. 14b). It is worth mentioning that the comparison shows only the probability of collapsed buildings as this is the only limit state provided by Jaiswal et al.'s model.

Apart from the noticeable differences in the identified building typologies, it can be observed that there is significant variability among the collapse rate functions between the vulnerability classes. In particular, it is important to note that the proposed fragility curves for poor (A) and medium (B) quality constructions in Malawi are more likely to collapse than according to the WHEPAGER poorest quality classifications (M2 and A). Furthermore, even good (C) quality buildings in Malawi have similar performance to one of the poorest classifications from WHE-PAGER, and they are far more likely to collapse than the WHE-PAGER classification that appears to have the closest description of the building type (UFB). Therefore, unfortunately, buildings in Malawi are far more vulnerable to earthquakes than WHE-PAGER suggests.

\subsection{Fragility functions for a weighted model}

Finally, the fragility curves for limit states are derived for all inspected façades. This allows defining fragility curves for a weighted model which takes into account the percentages of building typologies obtained from the structural surveys (i.e. $26 \%$ for A, $50 \%$ for B, and $24 \%$ for C) and related critical failure modes (i.e. $42 \%$ for OOP, $25 \%$
Table 4 Probability of exceeding the limit states by the proposed classification and WHE-PAGER classifications

\begin{tabular}{|c|c|c|c|c|}
\hline \multirow[t]{2}{*}{ LS } & \multicolumn{4}{|c|}{ Probability of exceeding LS at PGA (g) } \\
\hline & $0.1(\%)$ & $0.2(\%)$ & $0.3(\%)$ & $0.4(\%)$ \\
\hline \multicolumn{5}{|c|}{ Proposed weighted model } \\
\hline LD & 100 & 100 & 100 & 100 \\
\hline SD & $38-60$ & $83-87$ & 97-98 & 99-100 \\
\hline $\mathrm{NC}$ & $12-20$ & $58-64$ & $83-88$ & $96-98$ \\
\hline $\mathrm{C}$ & $10-19$ & $51-64$ & $77-87$ & $90-95$ \\
\hline \multicolumn{5}{|c|}{ WHE-PAGER weighted model } \\
\hline $\mathrm{C}$ & 4 & 18 & 28 & 32 \\
\hline
\end{tabular}

Only the probability of collapsed buildings is shown for WHEPAGER as this is the only limit state provided by this classification

for GABLE, 25\% for STRIP, and $8 \%$ for IP) distributed for each building typologies A, B and C as shown in Fig. 10a.

In Fig. 15, these fragility curves reported for the three behavioural models (i.e. geometric instability, limited ductility, and degradation of strength) are obtained using the fragility parameters of Table 7 . The curves show that the three behavioural models produce the same probability of predictions for LD, whereas the effects of the behavioural uncertainty can be significantly appreciated for $S D$, $\mathrm{NC}$ and $C$, as also reported in Table 4. Furthermore, it is also important to point out that for all three models the overall probability of collapse for all of the inspected buildings (which are a representative sample of all buildings in the area) is greater than $50 \%$ for a $P G A(\mathrm{~g})$ of 0.2 , confirming that non-engineered buildings in Malawi are extremely vulnerable to earthquakes of relatively low shaking intensity.

The probability of the collapsed buildings derived from the proposed weighted model is also compared with the weighted model obtained from the WHE-PAGER classifications, where for the latest the combined function is obtained by weighting the collapse rate functions of different vulnerability classes (i.e. M2, A, RS, UFB, and UCB) with respect to the individual percentages of buildings (Goda et al. 2016). The percentages of buildings assigned by Jaiswal and Wald (2008) to individual vulnerability classes of the WHE-PAGER classifications are $15 \%, 19 \%, 1 \%, 14 \%$, and $51 \%$, respectively. It is worth recalling that the comparison is only carried out for the probability of collapsed buildings as this is the only limit state provided by Jaiswal et al.'s model. 
Apart from the differences in the building typologies which were already discussed in Sect. 4.3.2, the percentages of buildings classified in them are also in significant disagreement, as reviewed in detail by Kloukinas et al. (2020). In addition to this, the curves give a very clear graphical representation of the differences in the proposed fragility curves (based on local data) from the ones provided by the WHE-PAGER classifications. These differences are also confirmed by the probability of collapsed buildings derived from WHE-PAGER, which are lower than the ones identified from the proposed model, as shown in Table 4. This reinforces an important conclusion: buildings in Malawi are much more vulnerable to earthquakes than previously assumed by the WHE-PAGER classifications.

\section{Conclusions}

The methodology presented in this paper is the first approach using a large sample of representative data from field surveys, extensive laboratory experiments, and analytical analyses, to assess the seismic vulnerability of nonengineered unreinforced masonry buildings in East Africa. Such a methodology was tested in Malawi, chosen as a representative country for its rapid expansion of informal settlements and construction practice in East Africa.

To this aim, procedures for on-site inspections for nonengineered buildings, and experimental tests to characterise the construction quality, geometric/structural features, and mechanical properties were introduced. A mechanical procedure was adopted to analyse the gathered data and estimate the critical failure modes of the surveyed houses which were approximated by equivalent single-degree-offreedom systems. To evaluate the structural performance, three behavioural models were proposed in this paper to capture the different performances and uncertainties which can be associated with a mechanical failure caused by geometric instability, limited ductility, and degradation of strength of the analysed systems. Finally, the results from the three behavioural models were employed to develop pushover curves, transformed into incremental dynamic curves for the derivation of seismic fragility functions.

The application of the proposed methodology on the non-engineered masonry buildings in Malawi has shown its feasibility in practice. Findings from the structural surveys and laboratory tests on local materials highlighted the important role played by different factors (i.e. masonry types, floor typologies, quality of constructions and mechanical responses) strongly affecting the vulnerability classes identified on the surveyed buildings and fragility functions. This has highlighted that it is important to derive fragility curves using vulnerability models capable of accounting for different parameters which express individual structural features preventing or promoting the activation of specific failure modes.

One important conclusion of this study is that nonengineered masonry buildings are highly vulnerable to earthquakes of low shaking intensity (i.e. for values of $P G A(g)$ equal to 0.2 and 0.3$)$. Furthermore, it was observed that the use of local data and rigorous analyses as described above predict significantly higher seismic vulnerability (damaged/collapsed) of buildings than estimated from previously available international reference data/models (e.g. WHE-PAGER classifications have vulnerability classes and building proportions for Malawi based on data inferred from Tanzania which differ significantly from the ones derived for the proposed procedure).

The fragility functions produced as a result of the proposed methodology reflect the seismic vulnerability of East Africa as well as the seismic vulnerability of most developing countries around the world, where non-engendered masonry building is the typical construction adopted for dwellings. These fragility curves can be derived for damage limit states and vulnerability classes following the proposed methodology or using the fragility parameters provided in "Appendix". As the fragility curves are calculated using the three behavioural models discussed above, three sets of fragility parameters are provided to define the behavioural uncertainty for each of the identified vulnerability classes.

Fragility parameters for a weighted model are also supplied. These are only valid for Malawi, as they are calculated with building percentages defined for the identified vulnerability classes through the structural surveys carried out for this study. This implies that to obtain combined fragility functions for other countries, the damage rate functions for vulnerability classes provided in "Appendix" need to be weighted with respect to the percentages of buildings representative for the area of study.

This work enhanced the knowledge of the built environment's vulnerability in developing countries and increased the awareness of the high seismic vulnerability for non-engineered masonry buildings. The derived fragi- 
lity curves could be considered as the first step for the development of a quantitative risk assessment tool and could serve to implement performance-based earthquake engineering methods in developing countries. In the future studies, it is recommended that such a methodology is also implemented to investigate possible low-cost retrofitting solutions to improve the building performance and reduce the seismic damage. This study could be used for making informed decisions in planning building regulation and policies.

\section{Appendix}

See Tables 5, 6 and 7 and Figs. 16, 17 and 18.
Table 6 Fragility parameters for building typologies

\begin{tabular}{llllllll}
\hline Class & Limit state & $\eta$ & $\beta$ & $\eta$ & $\beta$ & $\eta$ & $\beta$ \\
\hline A & LD & 0.03 & 0.33 & 0.03 & 0.33 & 0.03 & 0.33 \\
& SD & 0.05 & 0.38 & 0.08 & 0.32 & 0.06 & 0.32 \\
& NC & 0.15 & 0.39 & - & - & 0.13 & 0.34 \\
& C & 0.16 & 0.40 & 0.13 & 0.35 & 0.15 & 0.37 \\
B & LD & 0.03 & 0.27 & 0.03 & 0.27 & 0.03 & 0.27 \\
& SD & 0.08 & 0.40 & 0.11 & 0.34 & 0.06 & 0.34 \\
& NC & 0.16 & 0.43 & - & - & 0.15 & 0.38 \\
& C & 0.18 & 0.44 & 0.15 & 0.38 & 0.18 & 0.41 \\
C & LD & 0.05 & 0.38 & 0.05 & 0.38 & 0.05 & 0.38 \\
& SD & 0.09 & 0.68 & 0.14 & 0.56 & 0.11 & 0.60 \\
& NC & 0.25 & 0.41 & - & - & 0.24 & 0.39 \\
& C & 0.28 & 0.41 & 0.24 & 0.39 & 0.27 & 0.40 \\
& & & & & & &
\end{tabular}

${ }^{\mathrm{a}}$ Geometric instability

${ }^{\mathrm{b}}$ Limited ductility

${ }^{\mathrm{c}}$ Degradation of strength

Table 5 Fragility parameters for failure mode classes

\begin{tabular}{llllllll}
\hline Class & Limit state & $\eta$ & $\beta$ & $\eta$ & $\beta$ & $\eta$ & $\beta$ \\
\hline OOP & LD & 0.03 & 0.29 & 0.03 & 0.29 & 0.03 & 0.29 \\
& SD & 0.07 & 0.33 & 0.10 & 0.33 & 0.08 & 0.33 \\
& NC & 0.15 & 0.34 & - & - & 0.14 & 0.31 \\
& C & 0.16 & 0.36 & 0.14 & 0.31 & 0.16 & 0.34 \\
GABLE & LD & 0.04 & 0.30 & 0.04 & 0.30 & 0.04 & 0.30 \\
& SD & 0.05 & 0.42 & 0.08 & 0.36 & 0.06 & 0.36 \\
& NC & 0.17 & 0.45 & - & - & 0.14 & 0.40 \\
& C & 0.18 & 0.46 & 0.14 & 0.41 & 0.17 & 0.42 \\
STRIP & LD & 0.04 & 0.29 & 0.04 & 0.29 & 0.04 & 0.29 \\
& SD & 0.10 & 0.33 & 0.16 & 0.32 & 0.13 & 0.32 \\
& NC & 0.27 & 0.42 & - & - & 0.25 & 0.38 \\
& C & 0.29 & 0.43 & 0.25 & 0.39 & 0.29 & 0.41 \\
& LD & 0.08 & 0.26 & 0.08 & 0.26 & 0.08 & 0.26 \\
& SD & 0.44 & 0.22 & 0.44 & 0.22 & 0.44 & 0.22 \\
& NC & 0.48 & 0.19 & - & - & 0.48 & 0.19 \\
& C & 0.48 & 0.20 & 0.48 & 0.21 & 0.48 & 0.20 \\
& & & & & & &
\end{tabular}

Table 7 Fragility parameters for non-engineered masonry buildings (weighted model)

\begin{tabular}{|c|c|c|c|c|c|c|}
\hline Limit state & $\eta$ & $\beta$ & $\eta$ & $\beta$ & $\eta$ & $\beta$ \\
\hline \multicolumn{7}{|c|}{ Weighted model } \\
\hline LD & 0.04 & 0.38 & 0.04 & 0.38 & 0.04 & 0.38 \\
\hline SD & 0.08 & 0.68 & 0.12 & 0.56 & 0.10 & 0.60 \\
\hline $\mathrm{NC}$ & 0.18 & 0.55 & 0.11 & 0.51 & 0.17 & 0.52 \\
\hline $\mathrm{C}$ & 0.20 & 0.55 & 0.16 & 0.53 & 0.19 & 0.53 \\
\hline
\end{tabular}

${ }^{\mathrm{a}}$ Geometric instability

${ }^{\mathrm{b}}$ Limited ductility

${ }^{c}$ Degradation of strength 


\section{Fragility curves for Failure mode classes}

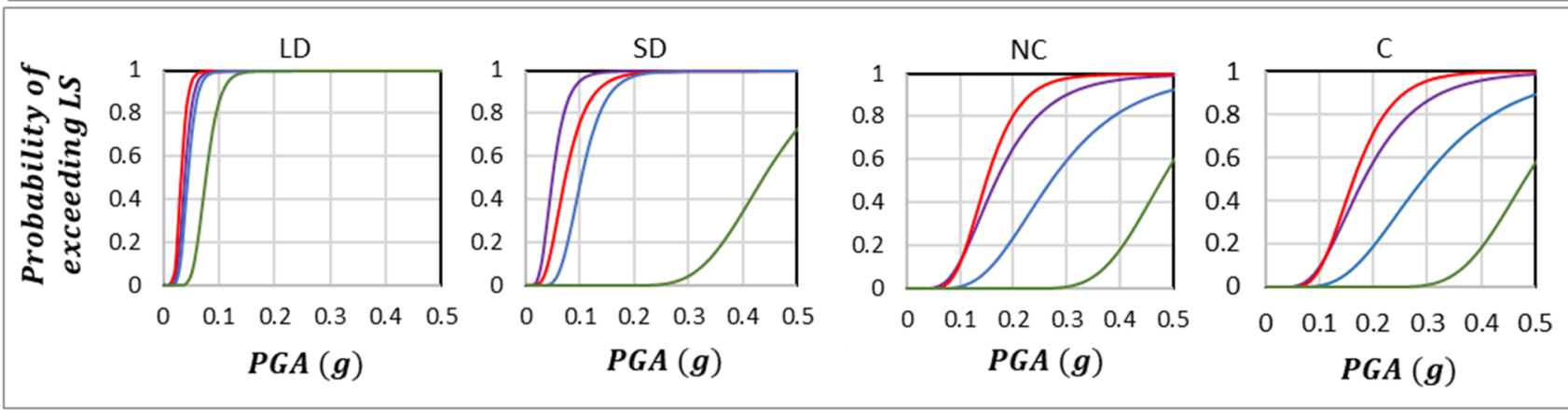

Geometric instability
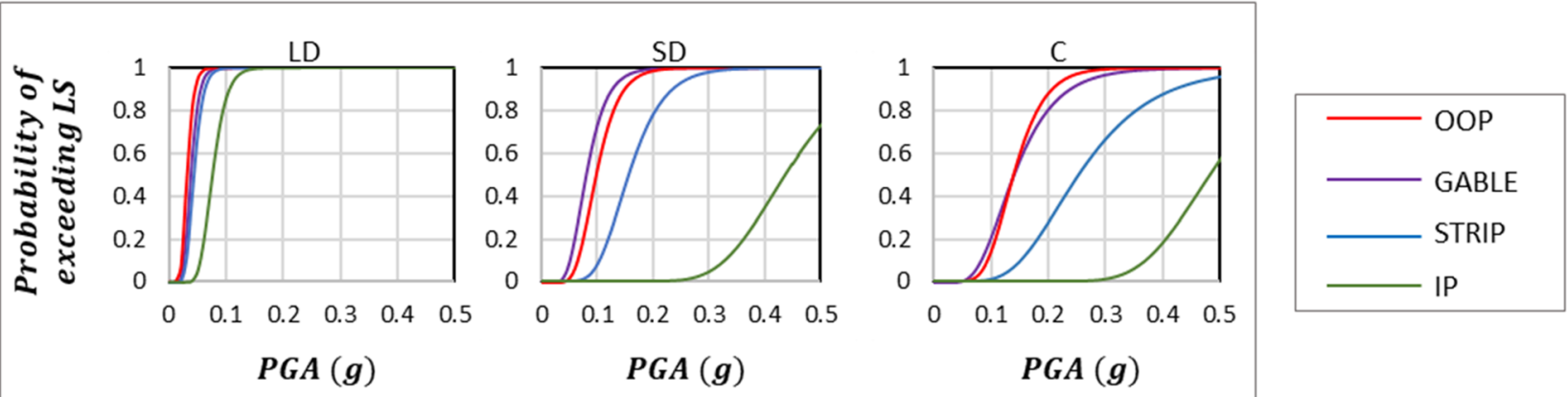

Limited Ductility

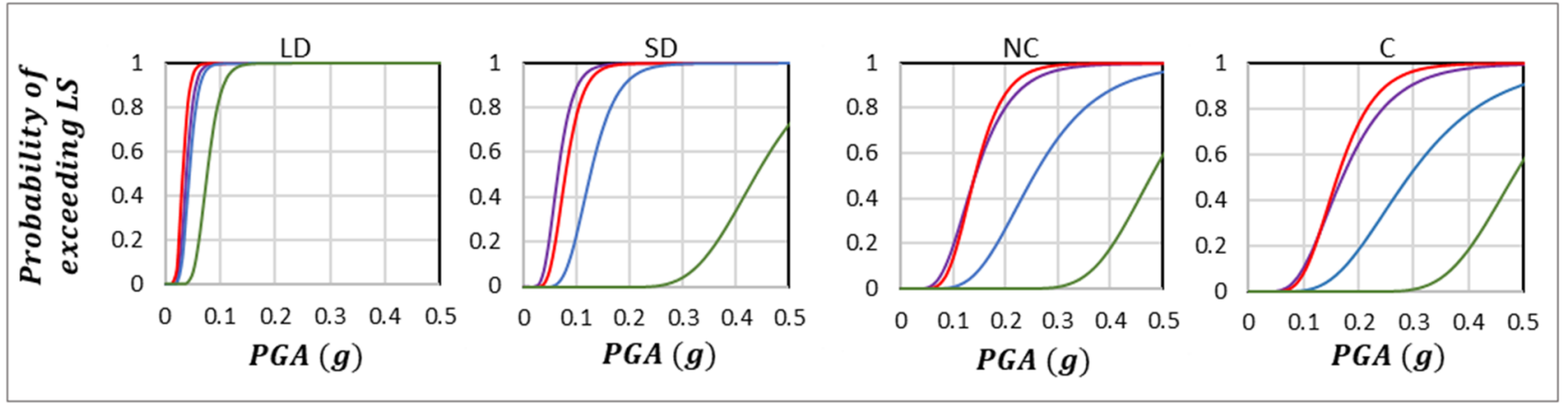

Degradation of strength

Fig. 16 Fragility curves for failure mode classes. Fragility curves are derived by Eq. 3 using $\eta$ and $\beta$ of Table 5 


\section{Fragility curves for building typologies}
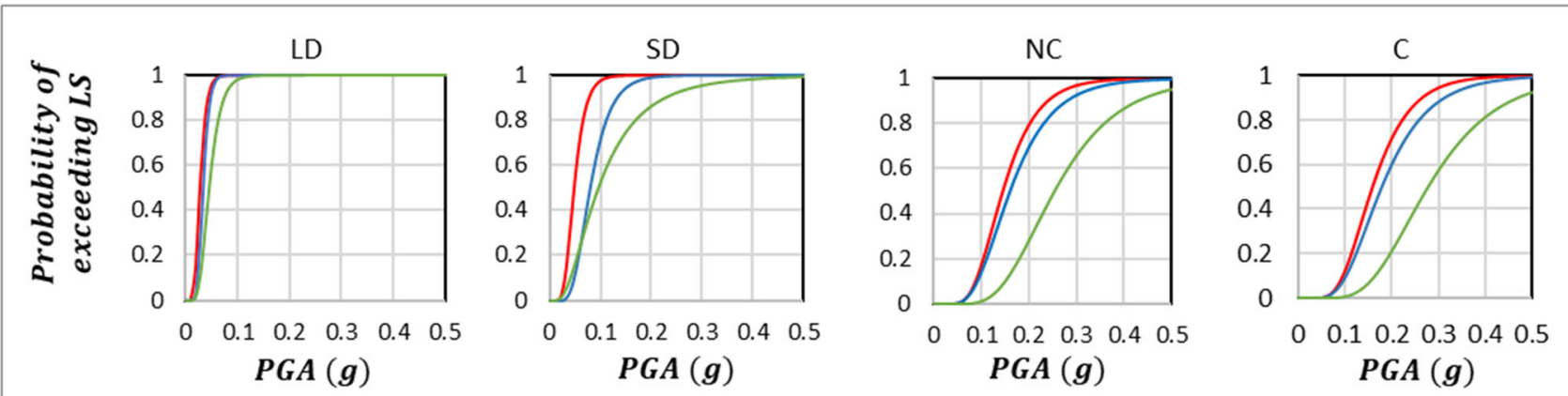

Geometric instability

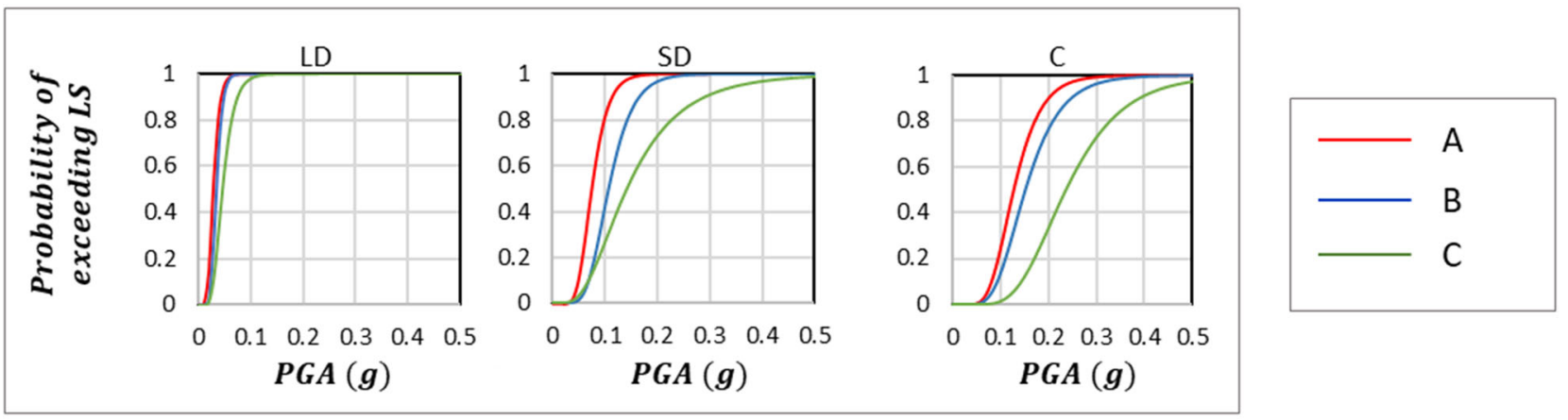

Limited Ductility

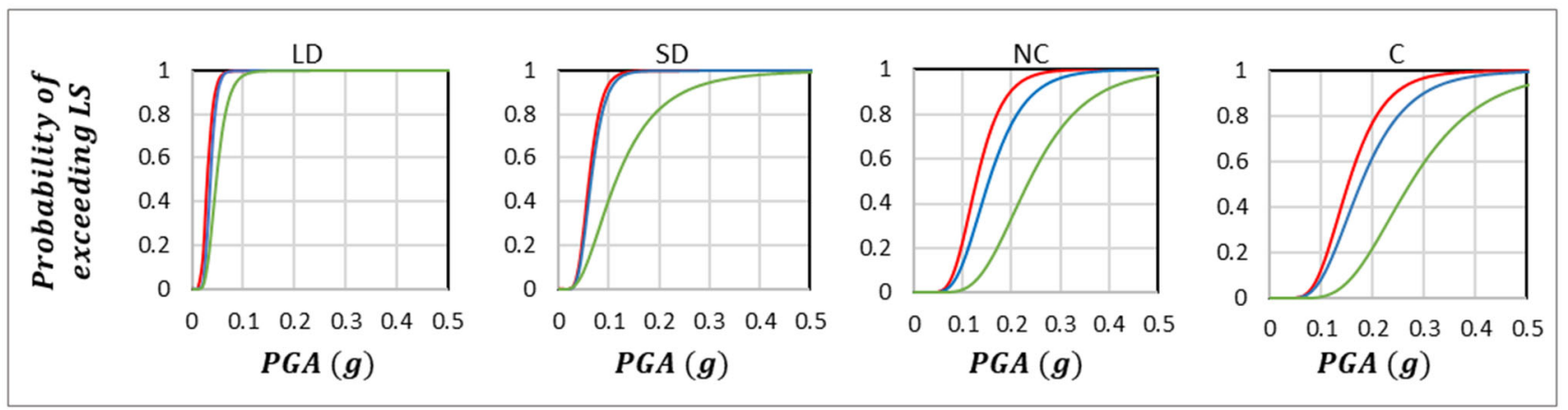

Degradation of strength

Fig. 17 Fragility curves for building typologies. Fragility curves are derived by Eq. 3 using $\eta$ and $\beta$ defined in Table 6 


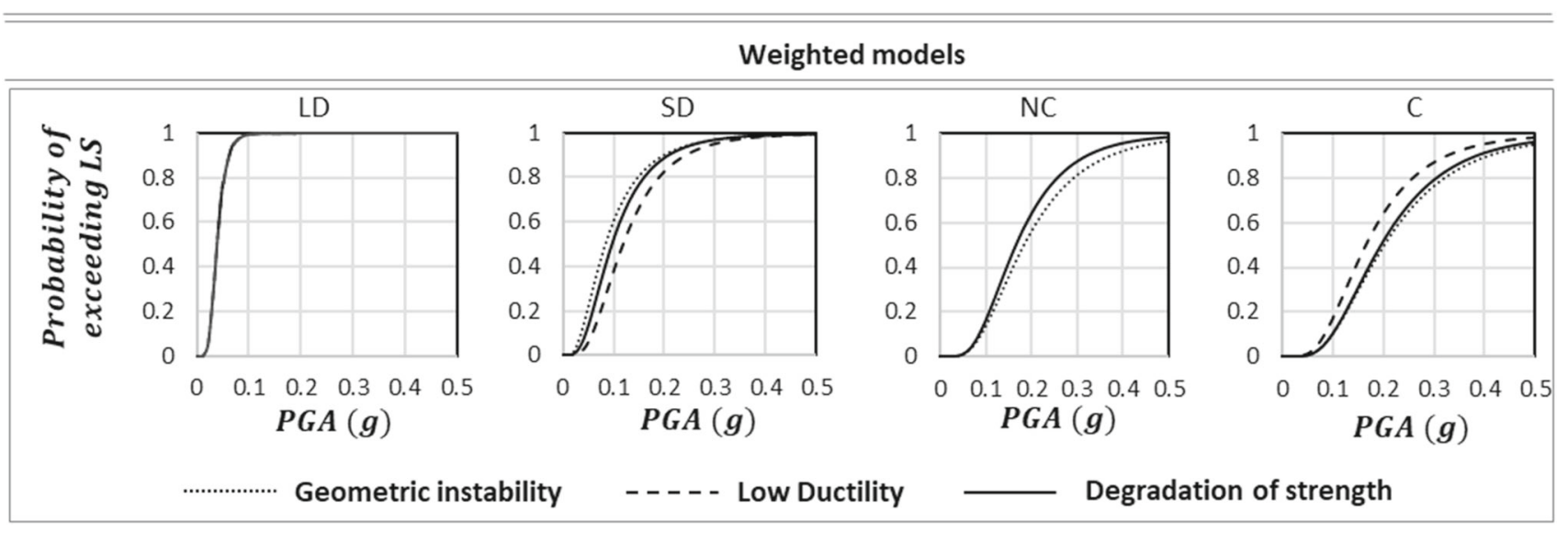

Fig. 18 Fragility curves for Non-engineering masonry buildings (weighted models). Fragility curves are derived by Eq. $3 \eta$ and $\beta$ defined in Table 7

List of symbols ADRS: Acceleration displacement response spectra; $T_{\text {eq }}$ : Natural vibration period of a SDOF system; $M_{\text {eff }}$ : Effective mass of a SDOF system involved in the failure mode; $E$ : Homogenised modulus of elasticity of the masonry; $I$ : Second moment of area; $\lambda$ : Collapse load factor multiplier; $t$ : Thickness of a façade; $L_{\text {tot }}$ : Total length of a façade; $H_{\text {tot }}$ : Total height of a façade; $s$ : Superposition between two bricks; $l$ : Brick length; $\mu$ : Ductility; SPO: Static pushover; SDOF: Single degree of freedom; LS: Limit states; LD: Light damage; SD: Severe damage; NC: Near collapse; $C$ : Collapse; $\mathrm{Sa}_{0}$ : Spectral acceleration at failure of a SDOF system; $\Delta_{\mathrm{c}}$ : Collapse displacement at failure of a SDOF system; $\mathrm{Sa}_{\mathrm{o}}-\Delta_{\mathrm{c}}$ : Bilinear relationship of a SDOF system at LD; $\Delta_{\mathrm{cr}}$ : Cracking displacement of a SDOF system at LD; $\mathrm{Sa}_{\mathrm{cr}}$ : Cracking spectral acceleration of a SDOF system at LD; $\delta_{\text {cr }}$ : Gradient of the linear system from the origin to LD (or SD). First branch of the SPO curve; $\Delta_{y}$ : Yielding displacement of a SDOF system at SD; $\mathrm{Sa}_{y}$ : Yielding spectral acceleration of a SDOF system at SD; $\Delta_{\mathrm{u}}$ : Ultimate displacement of a SDOF system at NC; $\mathrm{Sa}_{\mathrm{u}}$ : Ultimate spectral displacement of a SDOF system at NC; $\delta_{\mathrm{u}}$ : Gradient of the linear system from the origin to NC. If $\mu=1$ the linear system coincides with the first branch of the SPO curve or if $\mu \geq 1$ second branch of the SPO curve; $\Delta_{c^{*}}$ : Collapse displacement of a SDOF system at C; $\mathrm{Sa}_{c^{*}}$ : Collapse spectral acceleration of a SDOF system at C; $\mathrm{Sa}_{\mathrm{LS}}$ : Spectral acceleration at limit states; $\mathrm{Sa}_{0}^{\prime}$ : Spectral acceleration at the point intersection between the linear system with gradient $\delta_{\text {cr }}$ and the bi-linear $\mathrm{Sa}_{\mathrm{o}}-\Delta_{\mathrm{c}} ; \mathrm{Sa}_{\max }$ : Spectral acceleration at $\mathrm{SD}$, and $\mathrm{NC}$; PGA: Peak ground acceleration; GMPE: Ground motion prediction equation; $\widehat{\operatorname{PGA}(g)})_{\mathrm{GMPE}}$ : Average $\operatorname{PGA}(g)$; $\widehat{\operatorname{Sa} \mathrm{LS}_{\mathrm{GMPE}}}$ : Average spectral acceleration at limit sates; IDA: Incremental dynamic analysis; $P[\mathrm{LS} \mid \mathrm{PGA}]$ : Probably of exceeding a limit state for a given peak ground acceleration; $\Phi$ : Standard normal distribution function; $\eta$ : Equivalent median; $\beta$ : Logarithmic standard deviation

Acknowledgements The authors would like to thank the house owners and occupants in Balaka, Golomoti, Lifidzi, and Salima for their willingness to allow us to survey their homes.
Funding This study was funded by the Engineering and Physical Sciences Research Council through the PREPARE Project (EP/ P028233/1).

Data access statement Database of the collected data are available at the University of Bristol data repository.

\section{Compliance with ethical standards}

Conflict of interest The authors declare that they have no conflict of interest regarding the publication of this paper.

Ethical approval This article does not contain any studies with human participants performed by the authors.

Open Access This article is licensed under a Creative Commons Attribution 4.0 International License, which permits use, sharing, adaptation, distribution and reproduction in any medium or format, as long as you give appropriate credit to the original author(s) and the source, provide a link to the Creative Commons licence, and indicate if changes were made. The images or other third party material in this article are included in the article's Creative Commons licence, unless indicated otherwise in a credit line to the material. If material is not included in the article's Creative Commons licence and your intended use is not permitted by statutory regulation or exceeds the permitted use, you will need to obtain permission directly from the copyright holder. To view a copy of this licence, visit http://creativecommons. org/licenses/by/4.0/.

\section{References}

Addessi D, Marfia S, Sacco E, Toti J (2014) Modeling approaches for masonry structures. Open Civ Eng J 8(1):288-300

Baltzopoulos G, Baraschino R, Iervolino I, Vamvatsikos D (2017) SPO2FRAG: software for seismic fragility assessment based on static pushover. Bull Earthq Eng 15(10):4399-4425

Boore DM, Stewart JP, Seyhan E, Atkinson GM (2014) NGA-west 2 equations for predicting PGA, PGV, and 5\%-Damped PSA for shallow crustal earthquakes. Earthq Spect 30(3):1057-1085

Bureau TNM (2016) Safer house construction guidelines. https:// issuu.com/saferconstructionguidelines/docs/no-crocini 
Casapulla C, Argiento LU (2018) In-plane frictional resistances in dry block masonry walls and rocking-sliding failure modes revisited and experimentally validated. Compos B Eng 132:197-213

Chapola L, Gondwe J (2016) Urban development in earthquake prone areas: lessons from 1989 Salima and 2009 Karonga earthquakes. J Catholic Univ Malawi 2:15-26

Chopra AK (2000) Dynamics of structures: theory and applications to earthquake engineering. Prentice Hall, Upper Saddle River

D'Ayala D (2005) Force and displacement-based vulnerability assessment for traditional buildings. Bull Earthq Eng 3(3):235-265

D’Ayala D, Novelli V (2014) Seismic vulnerability assessment: masonry structures. Springer, Berlin

D'Ayala DF, Paganoni S (2011) Assessment and analysis of damage in L'Aquila historic city centre after 6th April 2009. Bull Earthq Eng 9(1):81-104

D'Ayala D, Speranza E (2003) Definition of collapse mechanisms and seismic vulnerability of historic masonry buildings. Earthq Spect 19(3):479-509

De Luca F, Verderame GM, Manfredi G (2015) Analytical versus observational fragilities: the case of Pettino (L'Aquila) damage data database. Bull Earthq Eng 13(4):1161-1181

De Luca F, Woods GE, Galasso C, D'Ayala D (2018) RC infilled building performance against the evidence of the 2016 EEFIT Central Italy post-earthquake reconnaissance mission: empirical fragilities and comparison with the FAST method. Bull Earthq Eng 16(7):2943-2969

Derakhshan H, Dizhur D, Griffith MC, Ingham JM (2014) In situ outof-plane testing of as-built and retrofitted unreinforced masonry walls. J Struct Eng 140(6):04014022

Diaz M, Zavala C, Flores E, Cardenas L (2019) Development of analytical models for confined masonry walls based on experimental results in Lima city. TECNIA 29(2):23-29

Doherty K, Griffith MC, Lam N, Wilson J (2002) Displacement-based seismic analysis for out-of-plane bending of unreinforced masonry walls. Earthq Eng Struct Dynam 31(4):833-850

Dumaru R, Rodrigues H, Varum H (2020) Seismic fragility assessment of revised MRT buildings considering typical construction changes. Front Struct Civ Eng 14:1-26

European Committee for Standardization (CEN) (2004) Design of structures for earthquake resistance. Part 1: general rules, seismic actions and rules for buildings. Eurocode 8

Federal Emergency Management Agency (FEMA) (1997) Guidelines and commentary for seismic rehabilitation of buildings. Reports nos. 273 and 274. October, Washington, DC

Federal Emergency Management Agency (FEMA) P-58-1 (2012) Seismic performance assessment of buildings. Volume 3supporting electronic materials and background documentation

Giordano N, De Luca F, Sextos A (2020) Out-of-plane closed-form solution for the seismic assessment of unreinforced masonry schools in Nepal. Eng Struct 203:109548

Goda K, Gibson ED, Smith HR, Biggs J, Hodge M (2016) Seismic risk assessment of urban and rural settlements around Lake Malawi. Front Built Environ 2:30

Griffith MC, Lam NT, Wilson JL, Doherty K (2004) Experimental investigation of unreinforced brick masonry walls in flexure. J Struct Eng 130(3):423-432

Hodge M, Biggs J, Fagereng Å, Mdala H, Wedmore LNJ, Williams JN (2020) Evidence from high-resolution topography for multiple earthquakes on high slip-to-length fault scarps: the BililaMtakataka Fault. Malawi. Tectonics 39(2):e2019TC005933

Imetrum Dynamic Monitoring (DMS). Bristol, UK. https://www. imetrum.com/

Kloukinas P, Kafodya I, Ngoma I, Novelli V, Macdonald J, Goda K (2019) Strength of materials and masonry structures in Malawi. In: Proceedings of the 7 th international conference on structural engineering, mechanics and computation (SEMC2019). 2-4 Sept 2019, Cape Town, South Africa

Kloukinas P, Novelli V, Kafodya I, Ngoma I, Macdonald J, Goda K (2020) A building classification scheme of housing stock in Malawi for earthquake risk assessment. J Housing Built Environ 35:507-537

Jaiswal KS, Wald DJ (2008) Creating a global building inventory for earthquake loss assessment and risk management (Open-File Report 2008-1160). US Geological Survey, Reston, p 103

Jaiswal KS, Wald DJ, D'Ayala D (2011) Developing empirical collapse fragility functions for global building types. Earthq Spect 27:775-795

Lagomarsino S (2015) Seismic assessment of rocking masonry structures. Bull Earthq Eng 13(1):97-128

Malawi Bureau of Standards Board (2014) The structural use of masonry-code of practice, part 1: unreinforced masonry walling, MS791

Moore A (2009) "Malawi earthquake relief". http://www.aaron moore.com.au/malawi-earthquake-relief\#/i/1

National Statistical Office of Malawi (2008) Population and housing census 2008. Zomba, Malawi: National Statistical Office. Retrieved from http://www.nsomalawi.mw/index.php

National Statistical Office of Malawi (2018). Preliminary report of 2018 population and housing census. Retrieved from http:// www.nsomalawi.mw/index.php

Novelli VI, D'Ayala D (2012) Assessment of the most damaged historic centres of the Region Emilia Romagna due to the earthquake of the 20th and 29th of May 2012. Ingegneria Sismica 29(2-3):59-71

Novelli VI, D’Ayala D, Makhloufi N, Benouar D, Zekagh A (2015) A procedure for the identification of the seismic vulnerability at territorial scale. Application to the Casbah of Algiers. Bull Earthq Eng 13(1):177-202

Novelli V, Kloukinas P, De Risi R, Kafodya I, Ngoma I, Macdonald J, Goda K (2019) Seismic mitigation framework for non-engineered masonry buildings in developing countries: application to Malawi in the East African rift. In: Resilient structures and infrastructure. Springer, Singapore, pp 195-223

Portioli F, Cascini L, Casapulla C, D' Aniello M (2013) Limit analysis of masonry walls by rigid block modelling with cracking units and cohesive joints using linear programming. Eng Struct 57:232-247

Preciado A, Ramirez-Gaytan A, Santos JC, Rodriguez O (2020) Seismic vulnerability assessment and reduction at a territorial scale on masonry and adobe housing by rapid vulnerability indicators: the case of Tlajomulco, Mexico. Int J Dis Risk Reduct 44:101425

Salmanpour AH, Mojsilovic N, Schwartz J (2013) Deformation capacity of unreinforced masonry walls subjected to in-plane loading: a state-of-the-art review. Int J Adv Struct Eng 5(1):22

Sorrentino L, D'Ayala D, de Felice G, Griffith MC, Lagomarsino S, Magenes G (2017) Review of out-of-plane seismic assessment techniques applied to existing masonry buildings. Int J Architect Herit 11(1):2-21

Tomaževič M (2007) Damage as a measure for earthquake-resistant design of masonry structures: Slovenian experience. Can J Civ Eng 34(11): 1403-1412

Vamvatsikos D, Cornell CA (2002) Incremental dynamic analysis. Earthq Eng Struct Dynam 31(3):491-514

Vamvatsikos D, Cornell CA (2006) Direct estimation of the seismic demand and capacity of oscillators with multi-linear static pushovers through IDA. Earthq Eng Struct Dynam 35(9): 1097-1117

Vlachakis G, Cervera M, Barbat GB, Saloustros S (2019) Out-ofplane seismic response and failure mechanism of masonry 
structures using finite elements with enhanced strain accuracy. Eng Fail Anal 97:534-555

World Bank Group, Report (2018) Malawi systematic country diagnostic: breaking the cycle of low growth and slow poverty reduction, 2018. No. 132785. World Bank Group, Washington, DC

\section{Authors and Affiliations}

\section{Viviana Iris Novelli ${ }^{1,2}$ (D) Raffaele De Risi $^{2} \cdot$ Ignasio Ngoma ${ }^{3} \cdot$ Innocent Kafodya ${ }^{3}$ Panos Kloukinas $^{4}$. John Macdonald ${ }^{2} \cdot$ Katsuichiro Goda ${ }^{5}$}

$\triangle$ Viviana Iris Novelli

NovelliV@cardiff.ac.ak; viviana.novelli@bristol.ac.uk

Raffaele De Risi

Raffaele.derisi@bristol.ac.uk

Ignasio Ngoma

Ingoma@ poly.ac.mw

Innocent Kafodya

Ikafodya@poly.ac.mw

Panos Kloukinas

P.kloukinas@greenwich.ac.uk

John Macdonald

John.Macdonald@bristol.ac.uk
Publisher's Note Springer Nature remains neutral with regard to jurisdictional claims in published maps and institutional affiliations.
Katsuichiro Goda

Kgoda2@uwo.ca

1 School of Engineering, Cardiff University, Cardiff, UK

2 Department of Civil Engineering, University of Bristol, Bristol, UK

3 University of Malawi, The Polytechnic, Blantyre, Malawi

4 University of Greenwich, Chatham, UK

5 Western University, London, Canada 